\title{
Axisymmetric Coulomb Interaction and Research of Its Stability by System Galactica
}

\author{
Joseph J. Smulsky \\ Institute of the Earth's Cryosphere, Siberian Branch of Russian Academy of Sciences, Tyumen, Russia \\ Email: jsmulsky@mail.ru
}

Received 3 July 2014; revised 9 August 2014; accepted 19 September 2014

Copyright (C) 2014 by author and OALib.

This work is licensed under the Creative Commons Attribution International License (CC BY). http://creativecommons.org/licenses/by/4.0/

(c) (i) Open Access

\section{Abstract}

Indeterministic consideration of micro-world based on quantum mechanics has resulted in its probabilistic understanding. At the same time the study of the micro-world based on Coulomb interactions in some cases gives deterministic view of it. However, at this way it is necessary to solve complex problems of many particles interaction. The program Galactica have to be developed for the numerical solution with high accuracy gravitational problems of many-bodies. The paper considers a modification of the program algorithm for solution of Coulomb interaction. For integrating differential equations of motion, the initial conditions have to be given, which are determined by the geometry of the interacting particles. Since in contemporary physics the atoms geometry is not specified, as an example, their axisymmetric models are studied. They consist of positively charged nucleus and are symmetrically arranged on the plane of the electrons. The necessary tasks were solved to determine positions and velocities of particles at the initial time. Based on their results, the program in environment MathCad for creation of a file of inital conditions is developed. Using the modified program Galactica, the motion of particles in an axisymmetric structure with eight peripheral electrons is researched. It has appeared that they are unstable. For comparison, a similar problem was studied with the gravitational interaction. It also proved to be unstable. So more detailed studies of the problem of stability of axisymmetric structures were made. They showed that the stability of the structure increases with the decrease of the interaction parameters. Such stable structure with eight peripheral bodies is considered for gravitational interaction. The paper also considers an example of a helium atom at axisymmetric interaction with two peripheral electrons. This structure is also unstable. At the same time two-particle interaction on the example of the hydrogen atom, considered using the Galactica, is stable and the results of numerical solutions coincide with the exact analytical solution. The studies showed that the program Galactica can be used to research the Coulomb interactions. The paper shows that axially symmetric structure of the atom can be used to create his other geometries. The developed methods and programs may be used in these studies. In the future, they will increase the degree of determinateness of micro-world. This paper, as well as the book, will be useful to physicists, students, senior pupils and everything who are interested in the scientific worldview. The programs are the free access (http://www.ikz.ru/ smulski/GalactcW/) and can be used for student projects. 


\title{
Keywords
}

\section{Coulomb Interactions, The Exact and Numerical Solutions, The Structure of the Atom}

\author{
Subject Areas: Functional Analysis, Mathematical Analysis
}

\section{1. Введение}

Для расчета движения при гравитационном взаимодействии многих тел разработан высокоточный метод интегрирования, который реализован в программе Galactica [1] [2]. На базе этой программы создана система свободного доступа Galactica [3], с помощью которой можно решать сложные задачи даже начинающему исследователю. При некоторой модификации исполняемого модуля системы Galactica она позволяет рассчитывать движение при кулоновском взаимодействии многих частиц.

Обычно в физике рассматривается кулоновское взаимодействие на основе задачи 2- $x$ частиц. А поведение ансамблей частиц и их свойства изучают в результате статистической обработки двухчастичных взаимодействий. Кулоновское взаимодействие многих частиц необходимо для развития планетарной модели атома. Однако в 20-м веке сложился индетерминированный подход к изучению микромира, при котором геометрия движений частиц не рассматривается, а свойства их ансамблей в квантовой механике описываются отвлеченными математическими функциями. Тем не менее, наряду с таким квантомеханическим рассмотрением микромира, рядом исследователей продолжает применяться классическая механика для объяснения ряда его явлений. Особенно много таких работ выполнено физикамидиссидентами. В Интернете можно найти даже подборки схем различных планетарных моделей атомов (см., например, [4]). А.Д. Власов [5] в своих исследованиях пришел к выводу о справедливости законов классической электродинамики внутри атома и несостоятельности их вероятностной интерпретации. Ф.М. Канарев на основе классической физики объясняет спектры излучения атомов. Правда он придерживается не орбитального взаимодействия электронов с протонами ядер, а линейного. Оно следует из выдвинутого им закона формирования спектров атомов и ионов [6] [7].

Особо следует отметить работы М. Грызинского. На протяжении нескольких десятилетий он настойчиво рассматривает явления микромира, основываясь на кулоновском механизме взаимодействия. Например, явление дифракции электронов М. Грызинский объясняет [8] прецессией спина электрона. Он считает, что такое объяснение закрывает корпускулярно-волновую природу частиц и открывает основу для детерминистского описания природных процессов. В работе [9] М. Грызинский на основе бинарных кулоновских взаимодействий рассматривает ионизацию атомов и молекул. При этом ему удается объяснить одинарную и двойную ионизацию; спектры излучения, например, излучение одной или триплетной линий; дифракцию частиц при их рассеянии на атомах и молекулах и т.д.

Открытым в 1921 г. эффектом К. Рамзауэра о слабом рассеянии электронов при малых их энергиях всегда объясняли неприменимость классической механики к явлениям микромира. М. Грызинский [10] [11] показал, что учет воздействия электронной оболочки атома позволяет объяснить это явление классическим кулоновским взаимодействием.

В работе [12] на основании классической механики он вывел уравнения для определения абсолютной энергии торможения частиц произвольной средой во всем нерелятивистском диапазоне энергий. Расчеты энергии торможения для конкретных веществ и частиц находятся в хорошем согласии с экспериментом.

Все эти задачи М. Грызинского имеют хорошее математическое обоснование. При этом он решает их аналитическими методами. Такой способ решения задач требует достаточно высокого уровня знаний исследователя не только в области физики, но и в области математики. Кроме того, даже при этих условиях решение задач взаимодействия многих частиц проблематично. Поэтому использование численных методов для решения таких задач кулоновского взаимодействия открывает перспективу детерминистского познания микромира.

\section{2. Дифференциальные Уравнения Движения При Кулоновском Взаимодействии}

Пусть имеется система материальных $N$ точек (частиц) с массами $m_{i}$ и электрическими зарядами $q_{i}$, 
где $i=1,2, \cdots, N$. Обозначим размерные координаты и скорости частицы $i$ как $x_{m i}, y_{m i}, z_{m i}, v_{x m i}$, $v_{y m i}, v_{z m i}$ в неускоренной системе координат с началом в центре масс C. В частности, координаты могут быть выражены в метрах, а скорости-в м/с. На частицу $i$ со стороны частицы $k$ оказывается электростатическое воздействие, которое в виде проекции силы Кулона на ось $x_{m}$ запишется так:

$$
F_{x i k}=\frac{q_{i} q_{k}\left(x_{m i}-x_{m k}\right)}{\varepsilon_{d} \cdot r_{m i k}^{3}},
$$

где $\varepsilon_{d}$-диэлектрическая проницаемость среды, в которой находятся частицы;

$$
r_{m i k}=\sqrt{\left(x_{m i}-x_{m k}\right)^{2}+\left(y_{m i}-y_{m k}\right)^{2}+\left(z_{m i}-z_{m k}\right)^{2}}
$$

расстояние между $i$-ой и $k$-ой частицами.

Выражение (1) для проекции сил на ось $x_{m}$ записано в системе единиц СГСЭ. Аналогичным образом выглядит выражение для силы в проекциях на оси $y_{m}$ и $z_{m}$. Поэтому здесь и в дальнейшем все выражения будем записывать для одной проекции. Отметим, что из соображений экономичности мы здесь не используем векторные, тензорные или матричные обозначения для записи общих выражений для силы.

Просуммировав силы (1) по всем частицам, получаем кулоновскую силу их воздействия на $i$-ую частицу

$$
F_{x i}=q_{i} \sum_{k \neq i}^{N} \frac{q_{k}\left(x_{m i}-x_{m k}\right)}{\varepsilon_{d} r_{m i k}^{3}},
$$

где знаком $\Sigma$ выражено суммирование по $k=1,2, \cdots, N$, за исключением $k=i$.

При воздействии (3) относительно неускоренной системы координат $i$-ая частица приобретает ускорение

$$
\frac{\mathrm{d} v_{m \times i}}{\mathrm{~d} t}=\frac{q_{i}}{m_{i}} \sum_{k \neq i}^{N} \frac{q_{k}\left(x_{m i}-x_{m k}\right)}{\varepsilon_{d} r_{m i k}^{3}} .
$$

Дальше задача решается в безразмерном виде. Для этого вводится характерный размер Am области, в которой находятся наэлектризованные частицы. Все заряды $q_{i}$ относим к абсолютной величине заряда электрона $e_{e}$, величина которого, например, в системе единиц СГСЭ $e_{e}=+4.80298 \times 10^{-10} \mathrm{~cm}^{3 / 2} \cdot \Gamma^{1 / 2} \cdot \mathrm{ce \kappa}^{-1}$. Массы $m_{i}$ относим к суммарной массе $M_{S S}$ всей системы взаимодействующих частиц. Тогда уравнение (4) в безразмерном виде примет следующий вид:

$$
\frac{\mathrm{d} v_{x i}}{\mathrm{~d} T}=-q m_{i} \cdot \sum_{k \neq i}^{N} \frac{q o_{k} \cdot\left(x_{i}-x_{k}\right)}{r_{i k}^{3}},
$$

где

$$
\begin{gathered}
x_{i}=x_{m i} / A m ; \quad r_{i k}=\sqrt{\left(x_{i}-x_{k}\right)^{2}+\left(y_{i}-y_{k}\right)^{2}+\left(z_{i}-z_{k}\right)^{2}} ; \\
q o_{i}=q_{i} / e_{e} ; \quad q m_{i}=-q o_{i} / m o_{i} ; \\
m o_{i}=m_{i} / M_{S S} ; \quad M_{S S}=\sum_{i=1}^{N} m_{i} ; \\
v_{x i}=v_{m x i} \cdot k_{v} ; \quad k_{v}=\sqrt{\frac{\varepsilon_{d} M_{S S} \cdot A m}{e_{e}^{2}}} ; \\
T=t \cdot k_{t} ; \quad k_{t}=\sqrt{\frac{e_{e}^{2}}{\varepsilon_{d} \cdot M_{S S} \cdot A m^{3}}}=1 /\left(A m \cdot k_{v}\right) .
\end{gathered}
$$

В этих уравнениях некоторые переменные: $q m_{i}, q o_{i}, m o_{i}, A m$ обозначены двумя буквами, чтобы в компьютерных программах и здесь обозначения были одинаковыми. 
Выражение (5) для трех проекций $x, y$ и $z$ представляет собой $3 \cdot N$ дифференциальных уравнений второго порядка, которые определяют движение заряженных частиц. Относительные заряды qо частиц могут быть положительные и отрицательные. Величина безразмерных единиц, которые описывают движение (5), зависит от произвольного параметра $A m$. Его значение можно выбрать таким, чтобы безразмерное время $T$ было в удобных единицах для рассмотрения взаимодействий в микромире.

Гравитационные взаимодействия в программе Galactica определяются следующим безразмерным уравнением [3]

$$
\frac{\mathrm{d} v_{x i}}{\mathrm{~d} T}=-\sum_{k \neq i}^{N} \frac{m o_{k}\left(x_{i}-x_{k}\right)}{r_{i k}^{3}} .
$$

Из сравнения (5) с (11) видно, что алгоритм для гравитационного взаимодействия можно приспособить к кулоновскому, если, во-первых, mo $_{k}$ заменить на qo ускорения $\mathrm{d} v_{x i} / \mathrm{d} T$ умножить на $q m_{i}$. Эти изменения были внесены в программу Galactica и создан исполняемый модуль glk3pb30 для кулоновского взаимодействия.

\section{3. Исходные Данные И Начальные Условия}

Для решения дифференциальных уравнений движения заряженных частиц (5) необходимо задавать исходные данные: $N, q m_{i}$ и $q o_{i}$ и начальные условия (НУ): координаты $x_{0 i}, y_{0 i}, z_{0 i}$ и скорости $v_{x 0 i}, v_{y 0 i}, v_{z 0 i}$ в начальный момент времени $T_{0}$. В качестве частиц рассмотрим 4 объекта: электрон, протон, нейтрон и ядро атома. Из этих объектов будем конструировать модели атомов. На основании данных справочника [13] (см. стр. 749, 807, 910, 912) принимаем массы электрона, протона и нейрона, соответственно: $m_{e}=9.1091 \times 10^{-31}$ кг; $m_{p}=1.67252 \times 10^{-27}$ кг; $m_{n e}=1.67482 \times 10^{-27}$ кг. Радиус ядра атома согласно [13] определяется выражением

$$
R_{n}=R_{0} \cdot A_{Z}^{1 / 3}
$$

где $R_{0}=1.5 \times 10^{-15} \mathrm{M}$;

$A_{Z}$-атомный вес, $Z$ - заряд атома.

Радиус электрона принимаем равным $R_{e}=1.5 \times 10^{-15}$ м.

Для определения радиуса орбиты электрона воспользуемся формулой для среднего расстояния электрона от ядра (см. стр. 749 [13]):

$$
r_{m}=\frac{a_{B o}}{2 \cdot Z}\left[3 \cdot n_{n}^{2}-l_{n}\left(l_{n}+1\right)\right],
$$

где $a_{\text {во }}=5.29167 \times 10^{-11}$ м-радиус первой боровской орбиты;

$n_{n}$ и $l_{n}$-квантовые числа.

В результате решений уравнений (5) с помощью программы Galactica была выбрана единица времени $P_{m}=10^{-15}$ сек, при которой безразмерные переменные $x, y, z$ и $T$ выражаются величинами чисел порядка нескольких десятичных знаков. Для преобразования размерных величин в безразмерные задается коэффициент времени в виде $k_{t}=1 / P_{m}$. Из выражения (10) через $k_{t}$ определяется характерный масштаб расстояния $A m$ так:

$$
A m=\left(\frac{10^{-9} \cdot e_{e}^{2}}{\varepsilon_{d} \cdot M_{S S} \cdot k_{t}^{2}}\right)^{1 / 3},
$$

где $10^{-9}$-коэффициент перевода единиц заряда в системе СГСЭ к единицам: м, кг и сек в системе СИ. Коэффициент скорости, в соответствии с (9), запишется так: $k_{v}=1 /\left(A m \cdot k_{t}\right)$.

Для определения начальных координат и скоростей частиц необходимо задавать геометрическую конфигурацию атома. Как уже отмечалось, в физике возобладал индетерминированный подход изучения микромира, поэтому в настоящее время конфигурация атома неизвестна. По-видимому, в результате решения рассматриваемых здесь задач она со временем определится. Ниже мы предприняли попытку рассмотрения плоской модели атома, которая состоит из ядра, осесимметрично расположенных $N_{1}$ электронов. Электроны обращаются вокруг ядра (см. Рис 1). Такая задача в случае гравитационного 
воздействия решена аналитически точно [1] [14]. Каждая из периферийных частиц движется вокруг центрального тела по траектории, которая в полярной системе координат $(r, \varphi)$ имеет вид:

$$
r_{i 1}=\frac{R_{p}}{\left(\alpha_{1}+1\right) \cos \left(\varphi-\varphi_{0 i}\right)-\alpha_{1}}, \quad i_{1}=1,2, \cdots, N_{1},
$$

где $a_{1}=m_{1} /\left(R_{p} \cdot v_{p}^{2}\right)$ 一параметр траектории;

$R_{p}$ - радиус перицентрия, т.е. наименьшее расстояние между траекторией и центральным телом;

$v_{p}$ - скорость тела в перицентрии;

$\varphi_{0 i 1}$ - начальный полярный угол $i_{1}$-ого тела в момент $T_{0}$.

$$
m_{1 g}=-G\left(m_{0}+m_{1} \cdot f_{n 1}\right)
$$

параметр взаимодействия; $G$-гравитационная постоянная; $m_{0}$-масса центрального тела; $m_{1}$-масса периферийного тела;

$$
f_{n 1}=0.25 \sum_{i_{2}=2}^{N_{1}} 1 / \sin \left[\left(i_{2}-1\right) \cdot \pi / N_{1}\right]
$$

коэффициент вклада воздействия $N_{1}$ периферийных тел.

Следует отметить, что параметр траектории $\alpha_{1}$ идентичен эксцентриситету $e$, и они связаны следующим выражением: $e=-\left(1+\alpha_{1}\right) / \alpha_{1}$. В дифференциальных уравнениях электромагнитного взаимодействия [1] параметр $\alpha_{1}$ играет аналогичную роль параметра траекторий, к которым понятие “эксцентриситет” неприменимо. Поэтому предпочтительно использовать параметр $\alpha_{1}$, а не эксцентриситет $e$.

В зависимости от параметра траектории периферийные частицы движутся по окружности $\left(\alpha_{1}=-1\right)$, по эллипсу $\left(-1<\alpha_{1}<-0.5\right)$, по параболе $\left(\alpha_{1}=-0.5\right)$, по гиперболе $\left(-0.5<\alpha_{1}<0\right)$ и по прямой $\left(\alpha_{1}=0\right)$.

Мы рассмотрели эту задачу при кулоновском взаимодействии: вокруг ядра с положительным зарядом $q_{0}=N_{1} e_{e}$ осесимметрично расположены на плоскости $N_{1}$ частиц с отрицательным зарядом $q_{1}=-e_{e}$. В этом случае параметр взаимодействия $\mu_{1}$ имеет вид:

$$
\mu_{1 e}=-\frac{10^{-9} \cdot e_{e}^{2}\left(N_{1}-f_{n 1}\right)}{m_{1} \cdot \varepsilon_{d}},
$$

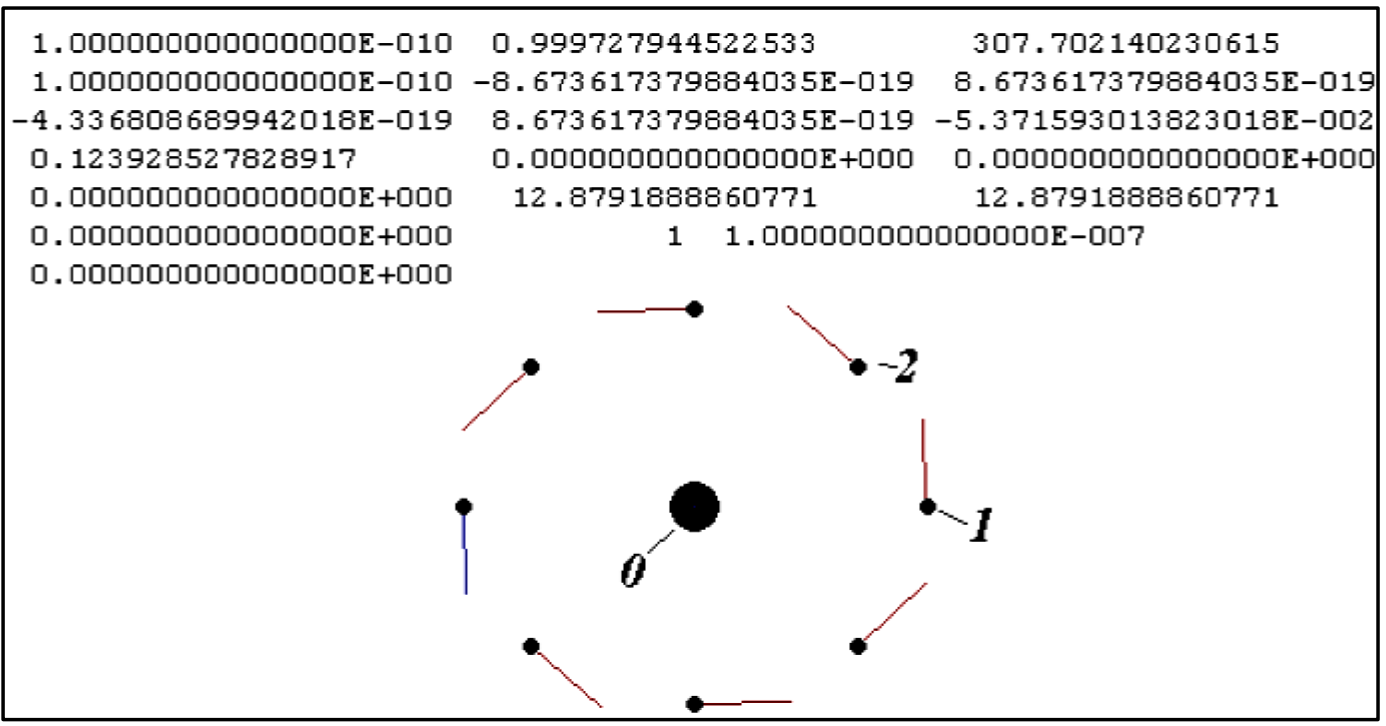

Рис 1. Осесимметричное кулоновское взаимодействие 9 частиц с параметрами для атома кислорода: 0-центральная частица; 1-первая периферийная частица; 2-вторая периферийная частица. Случай кругового движения. 
а все остальные свойства решения этой задачи такие же, как и в случае гравитационного взаимодействия. В формуле (18) кулоновское отталкивание между периферийными частицами отличается от гравитационного притяжения (16) знаком перед коэффициентом вклада воздействия $f_{n 1}$ периферийных частиц.

На основании решений этой задачи можно задать координаты и скорости частиц в осесимметричной модели атома. Введем плоскую систему координат $x_{o} O y_{o}$, в центре $O$ которой находится ядро, а на окружности радиусом $R_{p}$ равномерно расположено $N_{1}$ электронов. При этом первый электрон находится на оси $x_{o}$. Тогда координаты и скорости электронов в координатах $x_{o} y_{o}$ запишутся [15]:

$$
\begin{array}{ccc}
x_{o i 1}=R_{p} \cdot \cos \varphi_{o i 1} ; & y_{o i 1}=R_{p} \cdot \sin \varphi_{o i 1} ; & z_{o i 1}=0 ; \\
v_{o i 1}=-v_{p} \cdot \sin \varphi_{o i 1} ; & v_{y o i 1}=v_{p} \cdot \cos \varphi_{o i 1} ; & v_{z o i 1}=0
\end{array}
$$

где

$$
\begin{aligned}
& \varphi_{o i 1}=\left(i_{1}-1\right) \cdot 2 \pi / N_{1} ; \\
& v_{p}=\sqrt{\mu_{1} /\left(\alpha_{1} \cdot R_{p}\right)} .
\end{aligned}
$$

Начальные условия (19)-(20) зависят от двух параметров: радиуса перицентрия $R_{p}$ и параметра орбиты $\alpha_{1}$. Радиус перицентрия определяется через среднее расстояние $r_{m}$ электрона от ядра и параметра $\alpha_{1}$ так

$$
R_{p}=r_{m}\left(2 \alpha_{1}+1\right) / \alpha_{1} .
$$

Поэтому параметром $\alpha_{1}$ определяются начальные условия для электронов. Как уже отмечалось, для круговой орбиты $\alpha_{1}=-1$. Для расположенного в начале координат $O$ ядра координаты и скорости равны нулю.

Рассматриваемая осесимметричная задача является плоской. Из соображений общности результатов будем решать ее как пространственную в системе координат $x_{n} y_{n} z_{n}$. Пусть с ее осью $x_{n}$ совпадает ось $x_{o}$ орбитальной системы $x_{o} O y_{o}$. Вокруг оси $x_{n}$ система $x_{o} O y_{o}$ повернута на угол $\varepsilon_{0}$ относительно системы $x_{n} y_{n} z_{n}$. Тогда в размерном виде координаты частиц в системе координат $x_{n} y_{n} z_{n}$, начало которой $O$ находится в ядре, запишутся так

$$
x_{n i}=x_{o i} ; \quad y_{n i}=y_{o i} \cdot \cos \varepsilon_{o}-z_{o i} \cdot \sin \varepsilon_{o} ; \quad z_{n i}=y_{o i} \cdot \sin \varepsilon_{o}+z_{o i} \cdot \cos \varepsilon_{o} .
$$

Компоненты скорости $v_{x n i}, v_{y n i}$ и $v_{z n i}$ запишутся аналогично (24).

В программе Galactica предусмотрено более 10 способов контроля точности вычислений [16]. При превращении плоской задачи в пространственную добавляется еще один способ контроля точности, а именно, по изменению плоскости орбит рассматриваемых частиц.

Далее определяются координаты и скорости центра масс взаимодействующих частиц по известным формулам, которые для оси $x$ имеют вид:

$$
X_{c}=\sum_{i=1}^{N} m_{i} \cdot x_{n i} / M_{S S} ; \quad V_{x c}=\sum_{i=1}^{N} m_{i} \cdot v_{n i} / M_{S S},
$$

где $N=N_{1}+1$;

$$
M_{S S}=\sum_{i=1}^{N} m_{i}
$$

Следует отметить, что в рассмотренной осесимметричной конфигурации атома координаты и скорости цента масс равняются нулю. Поэтому отличие их от нуля будет характеризовать точность вычислений. Формулы (25) позволяют записать координаты и скорости частиц в безразмерном виде относительно центра масс, т.е. в неускоренной системе координат:

$$
x_{i}=\left(x_{n i}-X_{c}\right) / A m ; \quad v_{x i}=\left(v_{x n i}-V_{x c}\right) \cdot k_{v} .
$$

Выражения для проекций на оси $y \quad$ и z запишутся аналогично (27). Этими выражениями определяются НУ для интегрирования уравнений кулоновского взаимодействия (5). В рассмотренных ниже 
примерах угол $\varepsilon_{0}=0.409$ радиана, что соответствует наклону экватора Земли к плоскости ее орбиты. Поэтому графики в плоскости ху в дальнейшем будем называть графиками в экваториальной плоскости.

Рассмотренный алгоритм расчета исходных данных и НУ был реализован в среде МathСаd в виде программы InCnPrClb.mcd (см. Приложение). В результате работы программы создается файл исходных данных и начальных условий, например, с именем axsykc09.prn, который после некоторых изменений переименуется в файл axsykc09.dat. Последний файл используется для интегрирования уравнений (5) программой Galactica.

В пунктах программы InCnPrClb.mcd указаны основные этапы алгоритма. Кроме того, детальное описание аналогичной программы InCnPrpr.med для создания файла НУ при гравитационном взаимодействии дано в “Описании системы Galactica” на сайте http://www.ikz.ru/ smulski/GalactcW/, a также в работе [3].

\section{4. Осесимметричное Взаимодействие 9-И Частиц}

В качестве примера выбран атом кислорода с атомным весом $A_{Z}=16$, зарядом $Z=8$, количеством протонов, равным количеству нейтронов, т.е. по $Z$ частиц. Квантовые числа орбит электронов в формуле (13) заданы $n_{n}=2$ и $l_{n}=1$. Масса центральной частицы, т.е. ядра, будет $m_{o}=Z \cdot m_{p}+Z \cdot m_{n c}$. Общее число частиц в осесимметричной модели такого атома $N=9$, а число периферийных частиц, т.е. электронов, $N_{1}=8$. По представленному формулами (12)-(27) алгоритму были рассчитаны НУ. Все числовые значения для этого случая даны в программе InCnPrClb.mcd, приведенной в Приложении. $C$ подготовленным файлом НУ уравнения движения (5) были проинтегрированы с помощью программы Galactica.

Было рассмотрено две модели с эллиптическими орбитами электронов при эксцентриситете $e=0.15$ и с круговыми орбитами $(e=0)$. Менялся также шаг интегрирования $\Delta T=10^{-6} ; 10^{-7} ; 10^{-8}$. Задача решалась с двойной длиной числа (17 десятичных знаков) и с расширенной длиной (34 десятичных знака).

На Рис 1 показана несколько модифицированная выдача программы Galactica на экране монитора результатов этой задачи после первого шага счета с $\Delta T=10^{-7}$. Модификация заключается в изменении цвета изображения и направления вращения. Линиями у периферийных частиц представлены их вектора скорости, а числами даны время $T=1 \times 10^{-10}$; наибольшая масса $m_{o \max }=0.99972 \cdots$; модуль наибольшей скорости $v_{\max }=307.702 \cdots$; исполненный шаг счета $\Delta T_{p}=1 \times 10^{-10}$; проекции количества движения всей системы: $P_{x}, P_{y}, P_{z}$; проекции момента количества движения: $M_{x}, M_{y}, M_{z}$. Более детальное описание этих и семи следующих параметров задачи имеется в [3]. Последними двумя числами представлены величина следующего шага счета $\Delta T=10^{-7}$ и относительное изменение z-составляющей суммарного момента количества движения $\delta M_{z}=0$, где $\delta M_{z}=\left(M_{z}-M_{z 1}\right) / M_{z 1}, M_{z 1}$-момент количества движения системы на первом шаге счета.

Решение задачи можно наблюдать на экране монитора. После второго обращения периферийных частиц вокруг центральной частицы осесимметричная структура начинает изменяться и разрушается (см. Рис 2). На Рис 3 показаны проекции траекторий на плоскость ху центрального ядра (0), первого (1) и второго (2) электронов. Движение центрального ядра происходит за время $T=9.884 \times 10^{-2}$ в области размеров порядка $10^{-9}$, т.е. достаточно малой. Однако величина области непрерывно увеличивается. Траектории периферийных частиц 1 и 2 в течение 2-x обращений практически неизменны, а затем первая частица движется на периферию, а вторая-к центру.

На Рис 4 эти движения показаны на законах движения $x(T)$ этих частиц за время $T=0.2$. Здесь также линией 3 показано изменение расстояния $r$ первой частицы от начала координат. Решения на Рис 4 получены с расширенной длиной числа. Точность интегрирования уравнений (5) определяется [16] изменением кинетического момента системы $\delta M_{z}$. Если в решениях с двойной длиной числа величина $\delta M_{z}=1.64 \times 10^{-14}$, то с расширенной длиной- $\delta M_{z}=2.5 \times 10^{-25}$. То есть точность увеличилась на 11 порядков, а результаты до $T=0.09$ совпадают с результатами при двойной длине числа. В дальнейшем появляются различия этих решений: при двойной длине числа структура разрушается к концу третьего оборота (см. Рис 3), а при расширенной длине-в начале четвертого (см. Рис 4).

Итак, осесимметричная структура с 8-ю периферийными частицами при кулоновском взаимодействии разрушается после второго-третьего оборота. Осесимметричные структуры при гравитационном взаимо- 


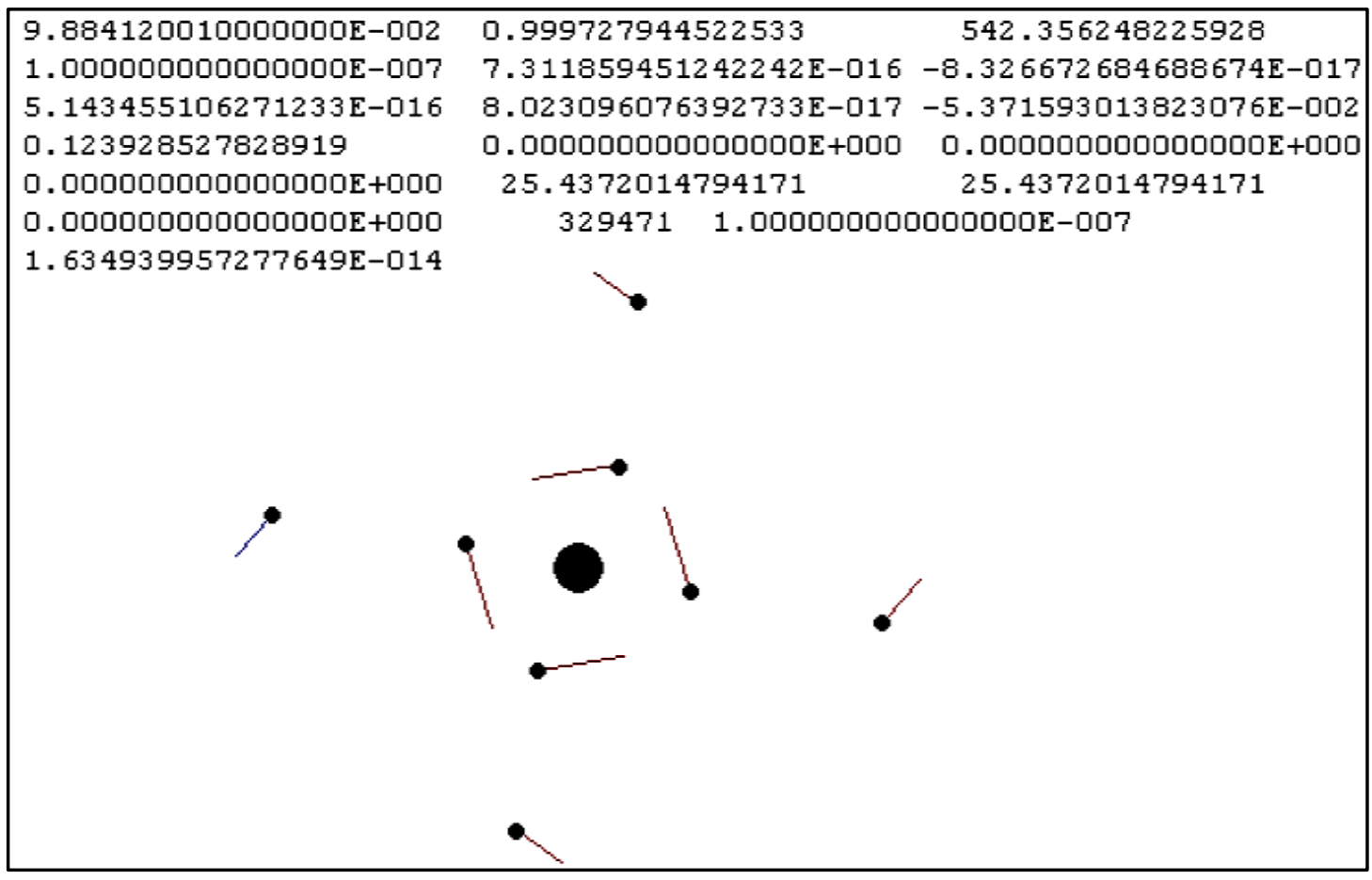

Рис 2. Вид на экране монитора сразу после разрушения кольца электронов осесимметричной структуры из девяти частиц. В начальном состоянии она показана на Рис 1.

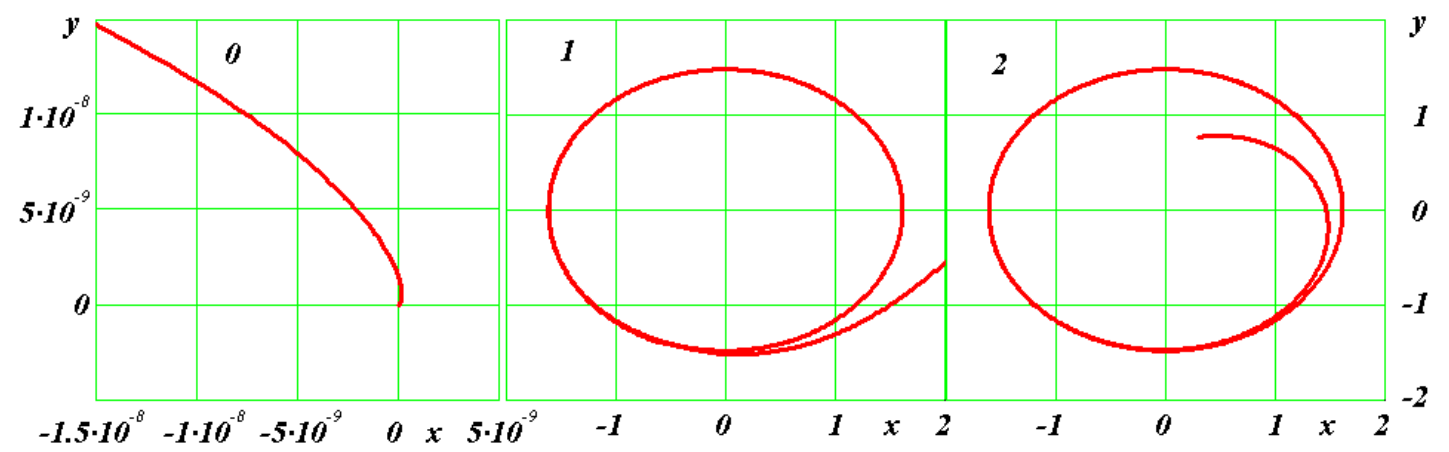

Рис 3. Траектории в экваториальной плоскости до момента $T=9.884 \times 10^{-2}: 0$-центральная частица; 1 первая периферийная частица; 2-вторая периферийная частица. Случай кругового движения.

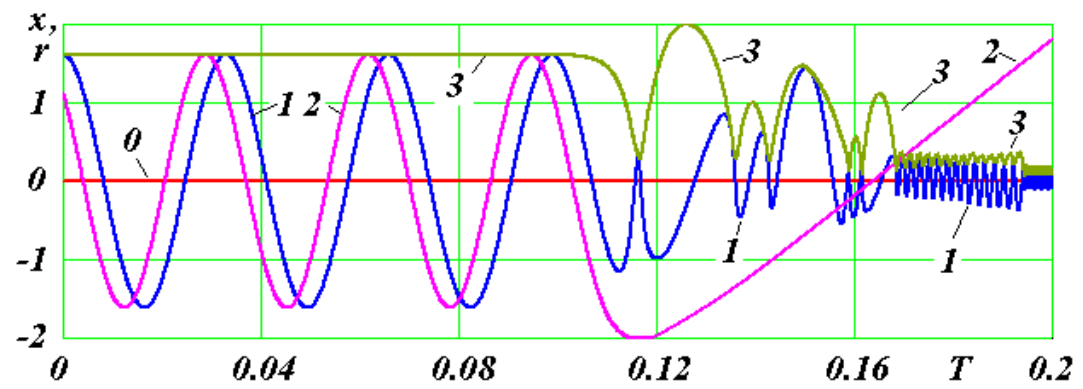

Рис 4. Изменение координаты $x$ во времени центральной частицы $(0)$ и двух периферийных частиц $(1,2)$, а также радиуса $r$ орбиты (3) первой частицы. Решение с расширенной длинной числа. Результаты до $T=0.09$ совпадают с решением при двойной длине числа. Осесимметричное кулоновское взаимодействие 9-и частиц с периодом $\bar{P}=0.0329$. 
действии рассматривались в качестве составных моделей Земли [17] и Солнца [18], а их эволюция изучалась на интервале до $T=1100$. Эти структуры не разрушались даже при наличии внешних тел, воздействующих на них. Поэтому возникла необходимость исследования гравитационной задачи, аналогичной рассмотренной кулоновской задачи.

\section{5. Аналогичная Задача С Гравитационным Взаимодействием}

Кулоновская задача (5) в безразмерных переменных (6)-(10) будет равнозначна гравитационной задаче (11), если в результате ее решения безразмерные параметры орбит будут одинаковы. В рассмотренной кулоновской задаче с параметрами для атома кислорода параметры орбиты в безразмерных единицах были следующие: полуось $\bar{a}=1.613$, период $\bar{P}=0.0329$ и скорость $\bar{v}=307.702$. Здесь безразмерные параметры, с целью их отличия от размерных, отмечены чертой сверху. Так как период для круговой орбиты рассчитывается через ее радиус $\bar{a}$ и скорость $\bar{v}$ по формуле $\bar{P}=2 \pi \bar{a} / \bar{v}$, то по существу орбиту определяют два параметра $\bar{a}$ и $\bar{v}$ или $\bar{a}$ и $\bar{P}$.

Зададимся целью создать задачу осесимметричного гравитационного взаимодействия с такими же параметрами $\bar{a}, \bar{P}, \bar{v}$ как и для кулоновского взаимодействия. Пусть суммарная масса взаимодействующих тел $M_{S S}$ будет задана. Из условия равенства рассмотренных параметров найдем массу $m_{1}$ периферийного тела. Так как для круговой орбиты параметр траектории $\alpha_{1}=-1$, то согласно (16) можно записать

$$
\alpha_{1}=\frac{\mu_{1 g}}{a v^{2}}=\frac{-G\left(m_{0}+m_{1} \cdot f_{n 1}\right)}{a v^{2}}=-1,
$$

откуда получаем выражение

$$
a v^{2}=G\left(m_{0}+m_{1} \cdot f_{n 1}\right) .
$$

Масштабный коэффициент скорости $k_{v}$ можно выразить [3] через масштаб расстояния $A_{m}$ :

$$
k_{v}=\sqrt{\frac{A_{m}}{G \cdot M_{S S}}}
$$

где масса всей системы

$$
M_{S S}=m_{0}+m_{1} \cdot N_{1}
$$

Выразим $а$ и $v$ через относительные величины: $a=\bar{a} \cdot A_{m}, \quad v=\frac{\bar{v}}{k_{v}}$.

После подстановки их в (29) имеем

$$
\bar{a} \cdot A_{m} \cdot \frac{\bar{v}^{2}}{k_{v}^{2}}=G\left(m_{0}+m_{1} \cdot f_{n 1}\right),
$$

или

$$
m_{0}+m_{1} \cdot f_{n 1}=M_{S S} \cdot \bar{a} \cdot \bar{v}^{2} .
$$

После подстановки $m_{0}$ из (31) в (32) получаем массу $m_{1}$ периферийного тела:

$$
m_{1}=\frac{M_{S S}\left(1-\bar{a} \cdot \bar{v}^{2}\right)}{N_{1}-f_{n 1}} \text {. }
$$

Так как в кулоновской задаче $\bar{a} \cdot \bar{v}^{2}=1.528 \times 10^{5}$, т.е. $\bar{a} \cdot \bar{v}^{2}>1$, а $f_{n 1}<N_{1}$, то получаем $m_{1}<0$, т.е. масса периферийных тел должна быть отрицательной. Таким образом, невозможно создать задачу осесимметричного гравитационного взаимодействия с такими же параметрами $\bar{a}, \bar{P}, \bar{v}$ как и для кулоновского взаимодействия.

Итак, получить с помощью гравитационного взаимодействия такое же относительное движение, как и при кулоновском, невозможно. При одинаковых полуосях орбит $\bar{a}$, скорость движения $\bar{v}$ по орбите при гравитационном взаимодействии должна быть значительно ниже. Поэтому было рассмотрено две 
гравитационных задачи. В первой были близкие по величине полуоси $\bar{a}$, а вовторой-одинаковые периоды $\bar{P}$. В этих двух задачах масса центрального тела $m_{0}$ и масса 8 периферийных тел равнялись массе Солнца $M_{S}=1.9891 \times 10^{30}$ кг. Таким образом: $M_{S S}=2 M_{S}=3.9782 \times 10^{30}$ кг, $m_{1}=M_{S} / N_{1}$.

В первой задаче была задана безразмерная полуось орбиты $\bar{a}_{1}=1.371$. По ней определяется размерный радиус орбиты $R_{p}$, по формуле (22)-скорость в перигелии $v_{p}$ и по формулам (19)-(20) рассчитаны начальные условия осесимметричной структуры. При этом относительные параметры получены следующие: $\bar{a}_{1}=1.371, \bar{v}_{1}=0.701, \bar{a}_{1} \cdot \bar{v}_{1}^{2}=0.675$ и период $\bar{P}_{1}=12.28$.

В этой гравитационной задаче, как и в кулоновской, структура является неустойчивой, и после 5 обращений (см. Рис 5) она разрушается. По сравнению с кулоновской задачей (см. Рис 4) тела до разрушения структуры совершают на два обращения больше. Кроме того, длительность ее существования по относительному времени $T$ почти в 500 раз больше, чем кулоновской структуры.

Во второй задаче была рассчитана величина полуоси $\bar{a}_{2}$, так чтобы период $\bar{P}_{2}$ совпал с периодом кулоновской задачи $\bar{P}=0.0329$. Для этого использовался третий закон Кеплера в следующей форме [1]:

$$
\frac{\bar{P}^{2}}{\bar{a}^{3}}=-\frac{4 \pi^{2}}{\alpha_{1}} .
$$

Из выражения (34) при одинаковом параметре траекторий $\alpha_{1}$ получаем величину полуоси $\bar{a}_{2}$ с таким же периодом $\bar{P}$ как и в кулоновской задаче

$$
\bar{a}_{2}=\left(\frac{\bar{P}^{2} \cdot \bar{a}_{1}^{3}}{\bar{P}_{1}^{2}}\right)^{1 / 3} \text {. }
$$

В этой задаче относительные параметры были $\bar{a}_{2}=0.02648 ; \bar{P}=0.0329, \bar{v}_{2}=5.05$ и $\bar{a}_{2} \cdot \bar{v}_{2}^{2}=0.675$. Если в первой задаче в абсолютных единицах радиус орбиты периферийных тел $a_{1}=126.7$ a.e. (астрономических единиц) и период обращения был равен $P_{1}=1228$ лет, то во второй задаче $a_{2}=2.448$ a.e. и $P_{2}=3.29$ года. Порядок скоростей движения в этих задачах такой же, как и в Солнечной системе, несмотря на то, что массы периферийных тел намного больше масс планет. А по сравнению с кулоновской задачей скорости значительно ниже.

Во второй гравитационной задаче 9-и тел (см. Рис 6) структура также неустойчива и разрушается к концу шестого обращения. По сравнению с кулоновской структурой она имеет в 50 раз меньшие относительные размеры. Длительность ее существования по относительному времени $T$ имеет такой же порядок, как и в кулоновской структуре.

\section{6. Проблема Устойчивости Осесимметричных Структур}

Во всех этих задачах было исследовано изменение $\Delta r$ радиуса орбит первого и вторых тел. Это изменение происходит одинаково. На Рис 7 оно показано на примере второй гравитационной задачи на трех разных пределах ординаты $\Delta r$ от $2 \times 10^{-14}$ до $4 \times 10^{-3}$. Здесь $\Delta r=r-r(0)$, где $r(0)$-радиус орбиты в начальный момент времени. По синусоиде 3 , период которой равен половине периода $\bar{P}$ обращения периферийных тел вокруг центрального тела, можно определять фазы отклонений тел 1 и 2 . Уже на первом обороте, как видно из графиков в пределах второго периода синусоиды 3, первое тело отклоняется к центру $(\Delta r<0)$, а соседнее тело 2 отклоняется от центра $(\Delta r>0)$. На втором обороте (в начале третьего периода синусоиды 3) тело 1 отклоняется от центра, а тело 2-к центру. При этом амплитуды отклонений возрастают. На первом полуобороте они были $\Delta r_{1}=-5 \times 10^{-15}$ и $\Delta r_{2}=1.7 \times 10^{-15}$, а на втором- $\Delta r_{1}=6 \times 10^{-14}$ и $\Delta r_{2}=-3.2 \times 10^{-14}$, т.е. возросли более чем на порядок. Такие колебания радиусов орбит соседних тел происходят с периодом, близким периоду обращения. По пересечениям линией 1 синусоиды 3 видно, что период этих отклонений постепенно увеличивается. Амплитуда колебаний с каждым обращением растет. На втором обороте они равны $\Delta r_{1}=1.7 \times 10^{-11} \quad$ и $\Delta r_{2}=-7 \times 10^{-12}$, т.е. увеличились по сравнению с первым оборотом больше чем в 200 раз. В таблице 1 приведены все последовательные отклонения радиусов орбит этих тел.

Как видно из Таблицы 1 на последнем 11-ом полуобороте отклонение первого тела достигает величины $\Delta r_{1}=-3.5 \times 10^{-3}$ и осесимметричная структура распадается. По сравнению с радиусом орбиты $r=2.648 \times 10^{-2}$ отклонение тела в этом случае достигает величины $\delta r_{1}=\Delta r_{1} / r=-0.132$. Поэтому ве- 


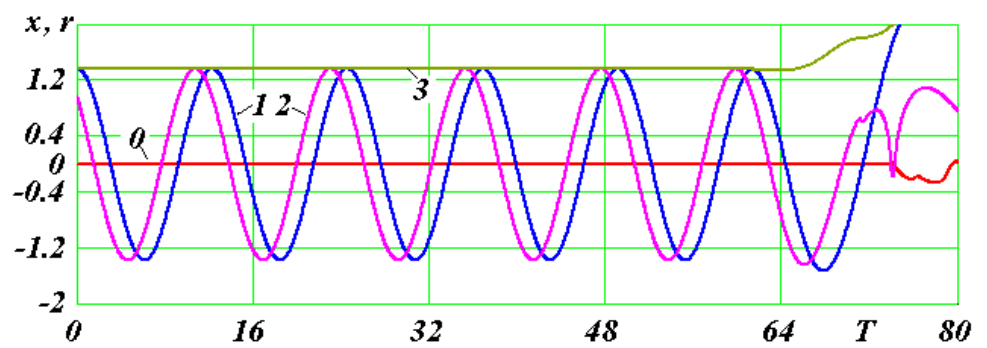

Рис 5. Осесимметричное гравитационное взаимодействие 9-и тел с периодом $\bar{P}=12.28$. Изменение во времени координаты $x$ центрального тела $(0)$ и двух периферийных тел $(1,2)$, а также радиуса $r$ орбиты (3) первого тела.

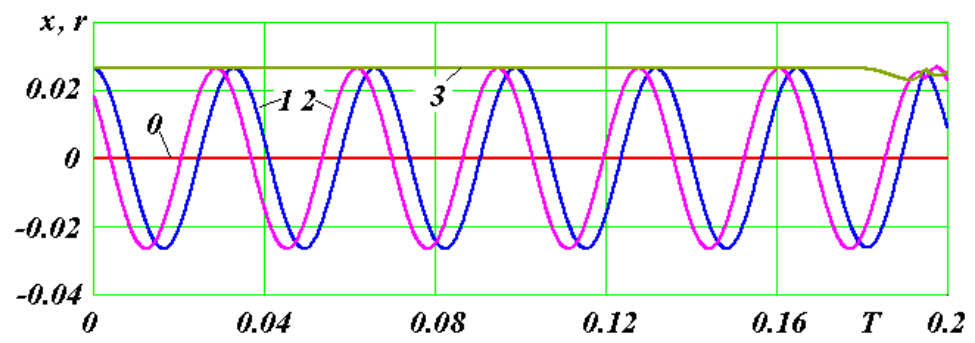

Рис 6. Осесимметричное гравитационное взаимодействие 9-и тел с периодом $\bar{P}=0.0329$. Изменение во времени координаты $x$ центрального тела $(0)$ и двух периферийных тел $(1,2)$, а также радиуса орбиты (3) первого тела.

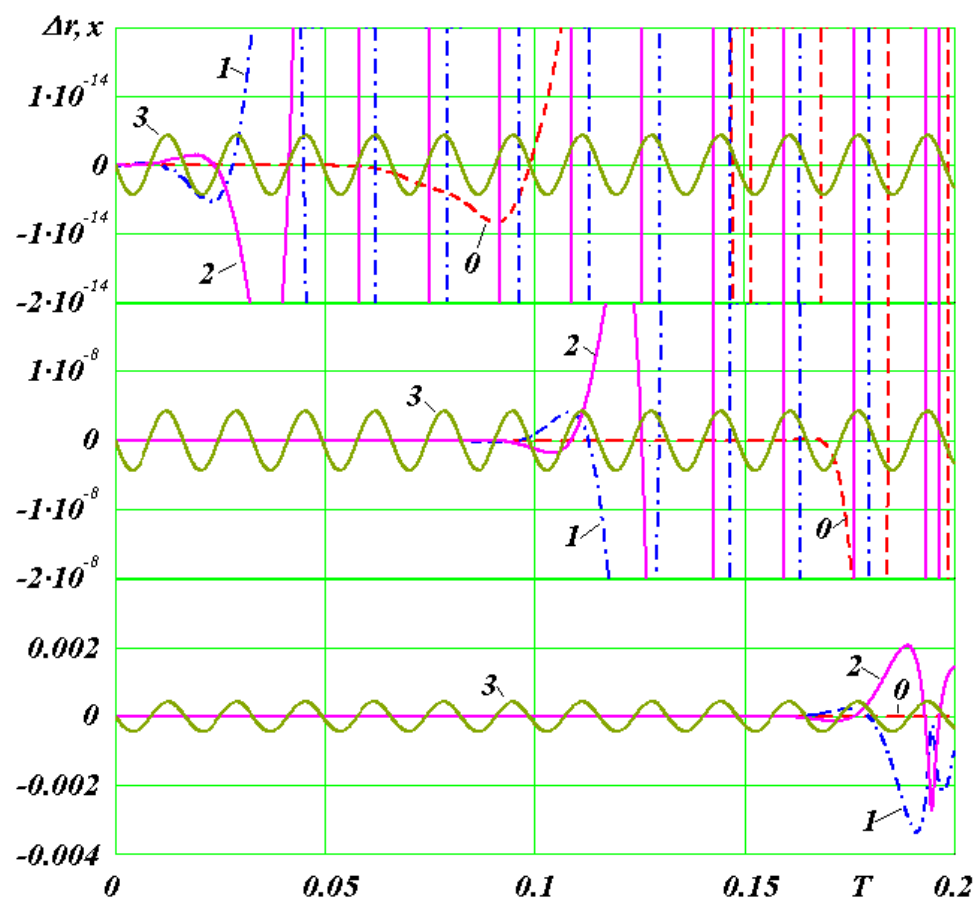

Рис 7. Осесимметричное гравитационное взаимодействие 9-и тел с периодом $\bar{P}=0.0329$. Изменение отклонения радиусов $\Delta r$ первого (1) и второго (2) тел, а также координаты $x$ центрального тела (0) при разных масштабах ординаты: 3 -синусоида с периодом $0.5 \cdot \bar{P}$. 
Таблица 1. Последовательные отклонения радиусов орбит первого $\left(\Delta r_{1}\right)$ и второго $\left(\Delta r_{2}\right)$ периферийных тел в осесимметричном гравитационном взаимодействии 9-и тел с периодом $\bar{P}=0.0329$.

\begin{tabular}{ccccccc}
\hline № п/п & 1 & 2 & 3 & 4 & 5 \\
\hline$\Delta r_{1}$ & $-5 \times 10^{-15}$ & $6 \times 10^{-14}$ & $-1 \times 10^{-12}$ & $1.7 \times 10^{-11}$ & $-2.5 \times 10^{-10}$ \\
$\Delta r_{2}$ & $1.7 \times 10^{-15}$ & $-3.2 \times 10^{-14}$ & $4.5 \times 10^{-13}$ & $-7 \times 10^{-12}$ & $1 \times 10^{-10}$ \\
\hline № ח п п & 6 & 7 & 8 & 9 & 10 & 11 \\
\hline$\Delta r_{1}$ & $4 \times 10^{-9}$ & $-7 \times 10^{-8}$ & $1 \times 10^{-6}$ & $-1.5 \times 10^{-5}$ & $2.4 \times 10^{-4}$ & $-3.5 \times 10^{-3}$ \\
$\Delta r_{2}$ & $-1.6 \times 10^{-9}$ & $3 \times 10^{-8}$ & $-5 \times 10^{-7}$ & $8 \times 10^{-6}$ & $-1.2 \times 10^{-4}$ & $2 \times 10^{-3}$ \\
\hline
\end{tabular}

личину отклонения радиуса орбиты периферийного тела порядка $\delta r=0.1$ можно считать пороговой для устойчивости этой осесимметричной структуры.

По изменению координаты $x$ центрального тела на Рис 7 видно, что оно также испытывает колебания. Первое отклонение его от центра наблюдается после второго обращения. Далее периоды отклонений уменьшаются. В конце рассмотренного интервала периоды отклонения центрального тела совпадают с периодами отклонения периферийных тел. При этом амплитуда колебаний центрального тела на четыре порядка меньше амплитуды колебания периферийных тел.

Такие же исследования изменений $\Delta r$ радиуса орбит первой и второй частиц были выполнены для кулоновского взаимодействия, рассмотренного на Рис 1-4. При гравитационном взаимодействии периферийные тела притягиваются, а при кулоновском взаимодействии периферийные частицы отталкиваются. Кроме того, кулоновское взаимодействие значительно сильнее гравитационного, и при нем орбитальная скорость на два порядка выше. На Рис 8 изменения $\Delta r$ радиуса орбит первой и второй частиц показано при одном пределе ординаты $\Delta r$, равном $4 \times 10^{-14}$. Линии отклонения радиусов первой $\left(\Delta r_{1}\right)$ и второй $\left(\Delta r_{2}\right)$ частиц имеются только в начале, т.е. радиусы орбит изменяются монотонно без колебаний. К моменту $T=0.1$, когда осесимметричная структура распадается, отклонения

$\Delta r_{1}=\Delta r_{2}=0.0048$. При радиусе орбиты $r=1.613$ относительное отклонение $\delta r=3 \times 10^{-2}$. Поэтому величину $\delta r \sim 0.01$ можно считать пороговой для устойчивости осесимметричной структуры. Она на порядок меньше по сравнению с $\delta r$ при гравитационном взаимодействии.

Из Рис 8 видно, что и центральная частица (0) при кулоновском взаимодействии совершает только одно колебание. Оно происходит после второго обращения, т.е. в момент разрушения структуры. При гравитационном взаимодействии, как видно из Рис 7, центральное тело колеблется также, как и периферийные тела.

\section{7. Устойчивая Осесимметричная Структура}

Как отмечалось ранее, осесимметричные структуры использовались для исследования эволюции составных моделей Земли [17] и Солнца [18]. Время их существования было неограниченным. Рассмотрим аналогичную структуру из 9-и тел, в которой масса центрального тела равняется массе Солнца $m_{0}=M_{S}$, а масса периферийных тел-массе всех планет, что составляет $1.342 \times 10^{-3} \cdot m_{0}$. В этой структуре относительные параметры были $\bar{a}=1.3625 \times 10^{-3}, \bar{v}=8.563, \bar{P}=9.997 \times 10^{-3}$ и $\bar{a}_{2} \cdot \bar{v}_{2}^{2}=0.1$.

Структура с приведенными параметрами была устойчивой, поэтому уравнения движения (5) интегрировались с шагом $d T=10^{-6}$, т.е. меньшим по сравнению с предыдущими решениями. Как видно из Рис 9, в течение 100 обращений координата $x$ первого и второго тела совершает гармонические колебания с периодом $\bar{P}$, а координата $x$ центрального тела и радиус орбиты $r$ остаются неизменными.

На Рис 10 в увеличенном масштабе ординаты показана динамика изменения радиусов $\Delta r$ орбит первого (1) и второго (2) тел, а также координаты $x$ центрального тела. В таком масштабе видны колебательные изменения радиусов орбит, которые имеют тенденцию к увеличению со временем. Амплитуда этих колебаний имеет порядок $1 \times 10^{-15}$. Точность вычислений определяется величиной изменения кинетического момента, которая в этом случае имеет величину $\delta M_{z}=1 \times 10^{-14}$. Поэтому колебания $\Delta r$ радиусов орбит периферийных тел обусловлены точностью вычислений и рассмотренная 


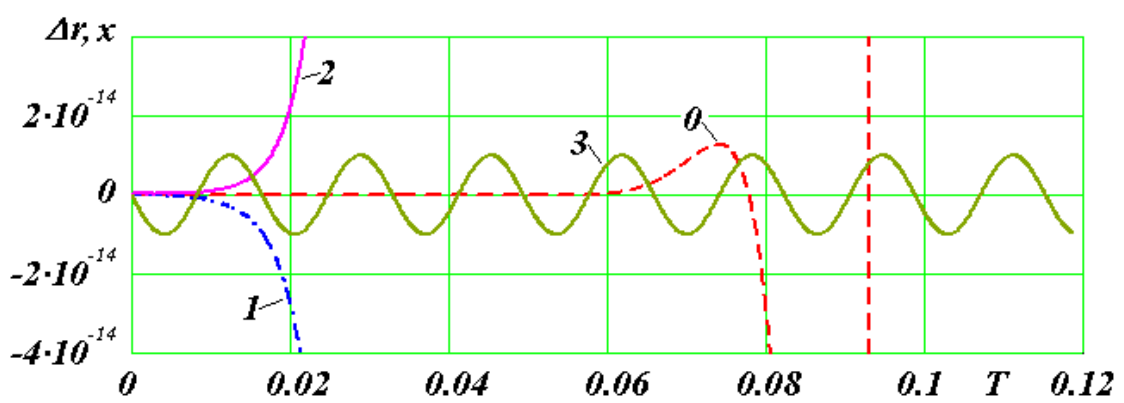

Рис 8. Осесимметричное кулоновское взаимодействие 9-и частиц с периодом $\bar{P}=0.0329$. Изменение отклонение радиусов $\Delta r$ первой (1) и второй (2) частиц, а также координаты $x$ центральной частицы $(0): 3$ - синусоида с периодом $0.5 \cdot \bar{P}$.

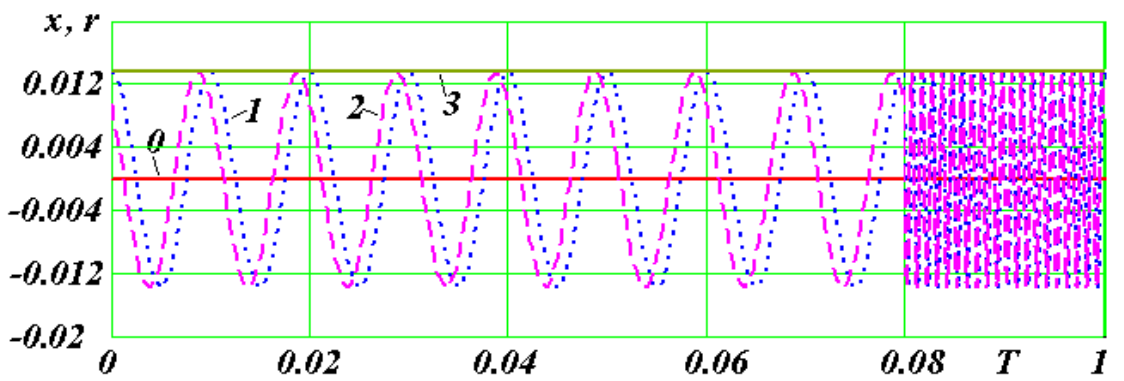

Рис 9. Устойчивое осесимметричное гравитационное взаимодействие 9-и тел с периодом $\bar{P}=9.997 \times 10^{-3}$. Изменение во времени координаты $x$ центрального тела $(0)$ и двух периферийных тел $(1,2)$, а также радиуса $r$ орбиты (3) первого тела. После $T=0.08$ масштаб времени изменен.

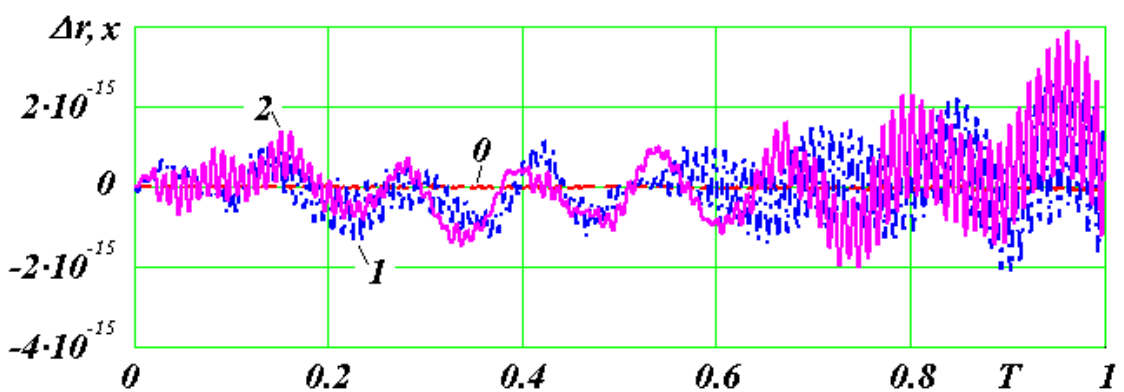

Рис 10. Изменение отклонение радиусов $\Delta r$ первого (1) и второго (2) тел, а также координаты $x$ центрального тела (0). Устойчивое осесимметричное гравитационное взаимодействие 9-и тел с периодом $\bar{P}=9.997 \times 10^{-3}$.

структура является устойчивой.

В формуле (33) масса $m_{1}$ периферийного тела при гравитационном взаимодействии определяется величиной $\bar{a} \cdot \bar{v}^{2}$. Покажем, что от нее зависит время существования осесимметричной структуры. В безразмерном виде это время будем определять в количестве оборотов структуры до ее распада. При $\bar{a} \cdot \bar{v}^{2}=1$ структуру невозможно создать, поэтому можно принять, что до распада она совершает 0 оборотов. В последнем случае устойчивой структуры условно примем число оборотов до распада равным одному миллиону. Тогда в четырех рассмотренных примерах величина $\bar{a} \cdot \bar{v}^{2}$ принимала значения: $1 ; 0.675 ; 0.675 ; 0.1$, а количество оборотов структур до распада были $0 ; 5 ; 5 ; 10^{6}$, т.е. с уменьшением величины $\bar{a} \cdot \bar{v}^{2}$ устойчивость структуры возрастает. В таком приближении можно считать, что осесимметричная структура будет устойчивой, если величина $\bar{a} \cdot \bar{v}^{2}<0.1$. 


\section{8. Осесимметричное Кулоновское Взаимодействие 3-х Частиц}

Ввиду неустойчивости кулоновского осесимметричного взаимодействия 9-и частиц была рассмотрена структура из трех частиц (см. Рис 11) на примере атома гелия с зарядом $Z=2$, атомным весом $A_{2}=4$ и квантовыми числами в формуле (13) $n_{n}=1, l_{n}=0$. При этих параметрах радиус ядра атома, согласно (12), $R_{n}=2.38 \times 10^{-15}$ м, а радиус орбиты осесимметрично расположенных двух электронов согласно (13) будет $r_{m}=3.969 \times 10^{-11}$ м. По этим данным файл НУ был подготовлен программой InCnPrClb.mcd, приведенной в Приложении. Относительные параметры орбиты в этом случае равны: $\bar{a}=1.2197$; $\bar{v}=102.7$ и $\bar{P}=7.462 \times 10^{-2}$.

При интегрировании уравнений (5) программой Galactica выяснилось, что структура разрушается после 8-ого оборота. На Рис 12 представлены траектории трех частиц. Центральная частица (0) с самого начала начинает движение по спирали с увеличивающимся радиусом. Первая частица после 8-ого обращения движется от центра, а вторая-к центру.

На Рис 13 эти движения продемонстрированы на законах изменения во времени координаты $x$ и радиуса $r$ орбиты первой частицы. На девятом обращении координата $x$ первой частицы становится больше полуоси орбиты $\bar{a}$, а второй частицы-не достигает $\bar{a}$. Как и в кулоновской структуре 9 частиц, в структуре с тремя частицами (см. Рис 14) отклонение радиусов первой $\left(\Delta r_{1}\right)$ и второй $\left(\Delta r_{2}\right)$ частиц увеличивается непрерывно без колебаний. К началу разрушения структуры $T=0.6$, отклонение радиусов орбит достигают значений $\Delta r_{1}=-\Delta r_{2}=0.0044$, что при радиусе орбиты $r=1.2197$ составляет относительную величину $\Delta r=3.6 \times 10^{-3}$. Это значение на порядок меньше величины $\delta r$ в осесимметричной кулоновской задаче 9-и частиц.

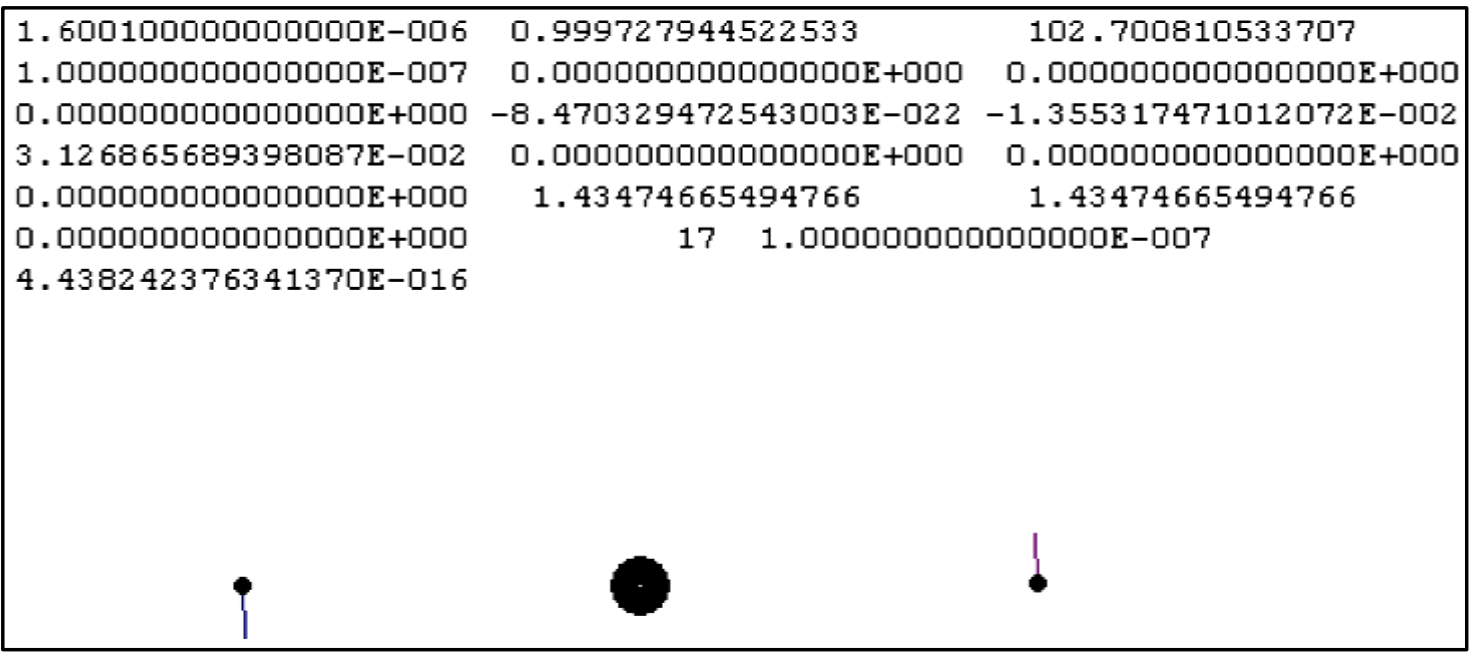

Рис 11. Осесимметричное кулоновское взаимодействие 3- $x$ частиц с параметрами для атома гелия.

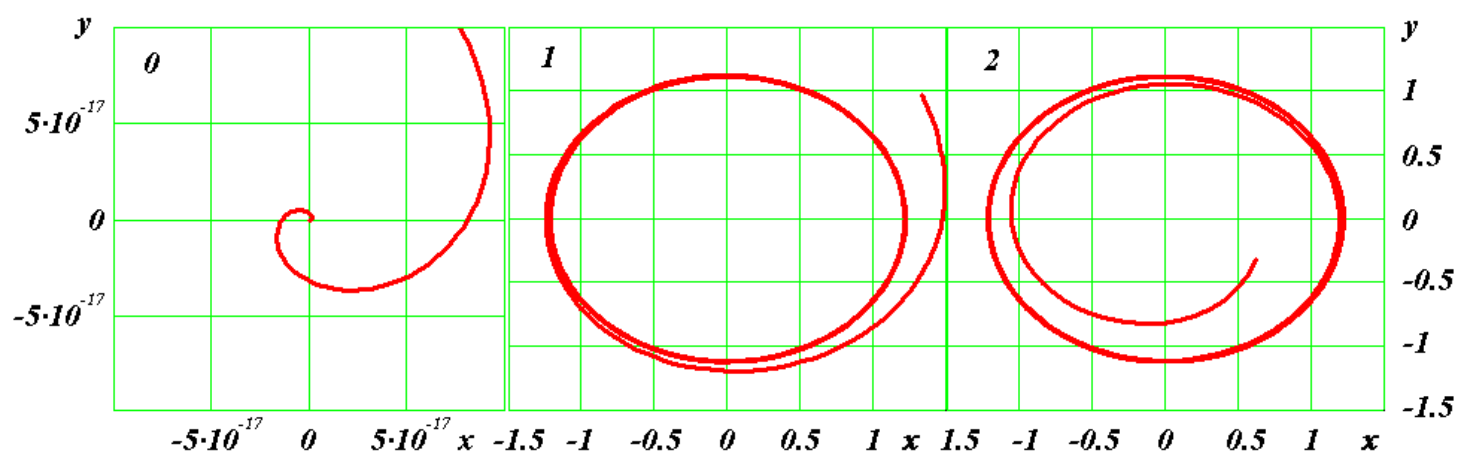

Рис 12. Осесимметричное кулоновское взаимодействие 3-x частиц. Траектории в экваториальной плоскости до момента $T=0.688$ : 0 -центральная частица; периферийные частицы: 1 -первая и 2-вторая. 


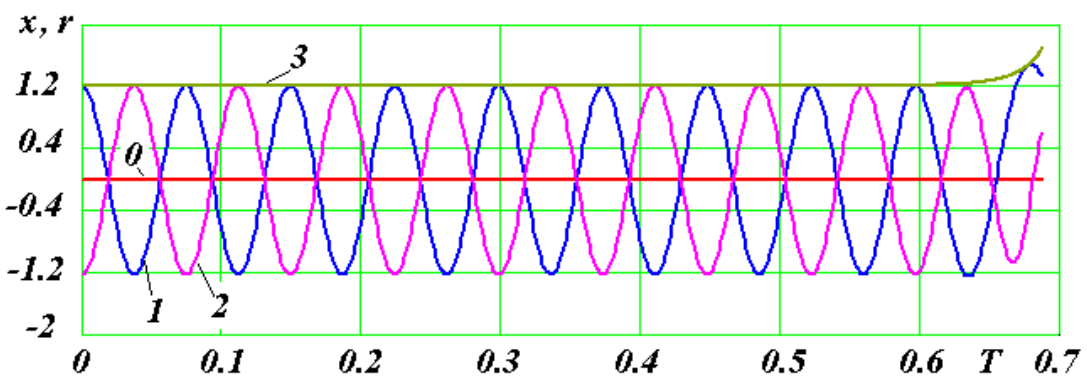

Рис 13. Изменение во времени координаты $x$ центральной частицы (0) и двух периферийных частиц $(1,2)$, а также радиуса орбиты (3) первой частицы. Осесимметричное кулоновское взаимодействие 3-x частиц.

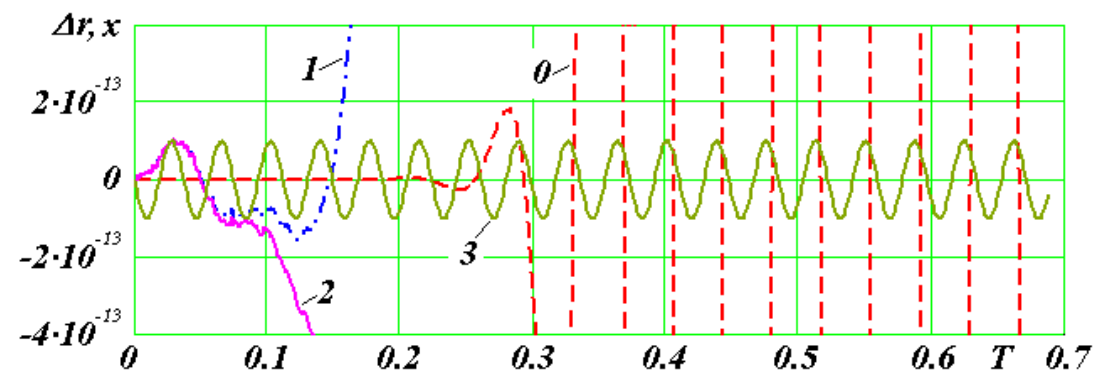

Рис 14. Изменение отклонения радиусов $\Delta r$ первой (1) и второй (2) частиц, а также координаты $x$ центральной частицы (0): 3 - синусоида с периодом $0.5 \times$ $\bar{P}$. Осесимметричное кулоновское взаимодействие $3-x$ частиц с периодом $\bar{P}=7.462 \times 10^{-2}$.

В кулоновской структуре из трех частиц центральная частица (0) на Рис 14 совершает колебания с увеличивающейся амплитудой. Период их равен периоду обращения. Эти колебания подобны колебаниям центрального тела в гравитационной задаче 9-и тел (см. Рис 7).

\section{9. Кулоновское Взаимодействие 2-х Частиц}

Динамика осесимметричных кулоновских структур была исследована с помощью программы Galactica. Они оказались неустойчивыми. Поэтому представляет интерес решение этой программой задачи кулоновского взаимодействия двух частиц, которая также имеет точное аналитическое решение. Однако в этой задаче отсутствуют причины для изменения орбиты. Задача 2-x частиц (см. Рис 15) рассматривалась на примере атома водорода с зарядом $Z=1$, атомным весом $A_{2}=1$ и квантовыми числами в формуле (13) $n_{n}=1, l_{n}=0$. При этих параметрах радиус ядра атома согласно (12) $R_{n}=1.5 \times 10^{-15} \mathrm{M}$, а радиус орбиты электрона согласно (13) будет $r_{m}=7.938 \times 10^{-11}$ м. По этим данным файл НУ также подготовлен программой InCnPrClb.mcd, приведенной в Приложении. Однако, т.к. задача двух частиц не является осесимметричной, то параметр взаимодействия $\mu_{1}$ в пункте 14.5 рассчитывается в соответствии с пунктом 14.14 .

Относительные параметры орбиты в этом случае равны: $\bar{a}=1.5365 ; \bar{v}=34.56$ и $\bar{P}=0.2791$. Динамика этой структуры является устойчивой. Это видно по графической выдаче программы Galactica (см. Рис 15) после 1000 обращений. Траектории частиц за 100 обращений показаны на Рис 16 десятью точками на одно обращение. Эти точки, сливаясь за 100 обращений, образуют отрезки линий. Радиус орбиты ядра (0) примерно в 2000 раз меньше радиуса орбиты электрона.

На Рис 17 представлены законы изменения координаты $x(T)$ центральной (0) и периферийной (1) частиц на десяти начальных и десяти конечных обращениях частицы. Точками 3 показано точное аналитическое решение задачи. Как видно из графиков, численные решения не отличаются от него.

На Рис 18 показана динамика отклонения радиуса $\Delta r$ орбиты периферийной частицы. Величина $\Delta r$ изменяется колебательно и за 100 обращений амплитуда достигает значения $\Delta r=5 \times 10^{-12}$, что со- 


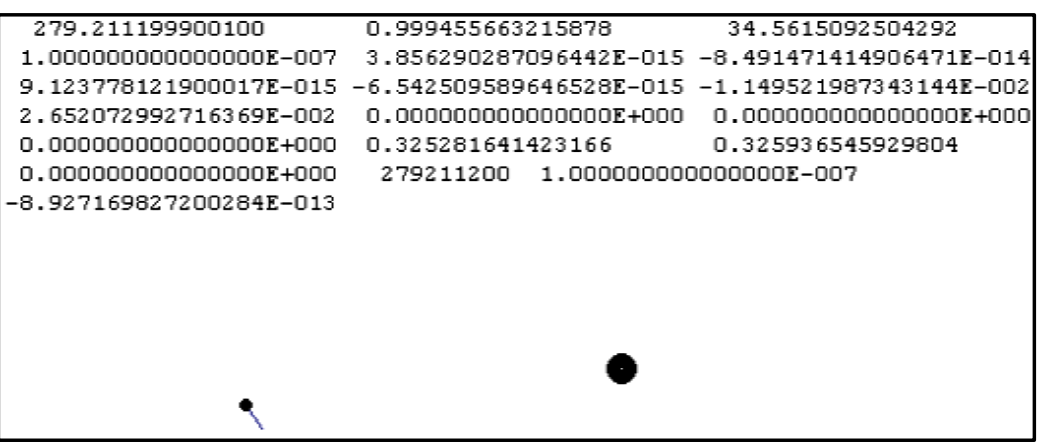

Рис 15. Кулоновское взаимодействие 2-x частиц с параметрами для атома водорода после 1000 обращений. Период обращения $\bar{P}=0.2791$.

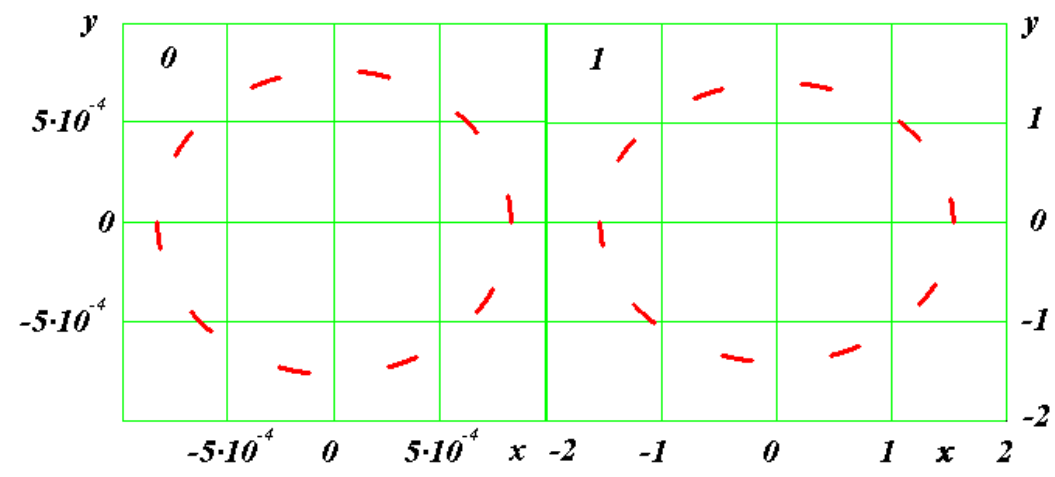

Рис 16. Траектории в экваториальной плоскости за 100 обращений до момента $T=27.92: 0$-центральная частица; 1 -периферийная частица. Траектории показаны 10 точками на одно обращение.

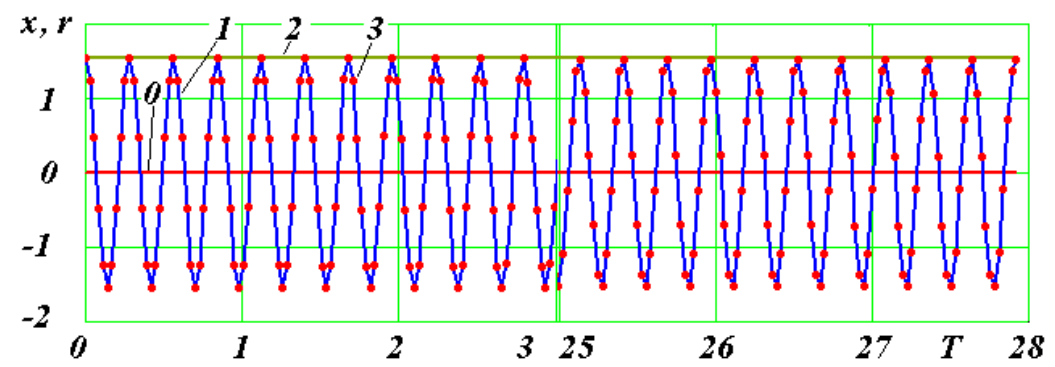

Рис 17. Изменение во времени координаты $x$ центральной частицы (0) и периферийной частицы (1), а также радиуса орбиты (2) периферийной частицы на двух интервалах времени $T: 0$ - 3 и 25 - 28. 3-точное аналитическое решение.

ставляет относительное изменение $\delta r=3 \times 10^{-12}$. В этом случае величина погрешности $\delta M_{z}=4.8^{-13}$. Эта задача была проинтегрирована с расширенной длиной числа с шагами $\delta T=10^{-7}$ и $10^{-8}$, при которых относительные изменения кинетического момента были соответственно $\delta M_{z}=2.03 \times 10^{-28}$ и $2.87 \times 10^{-30}$. В такой же пропорции уменьшается величина отклонения радиуса орбиты $\delta r$. Таким образом, увеличение отклонения радиуса $\Delta r$ на Рис 18 обусловлено погрешностью решения задачи.

Итак, численное решение программой Galactica кулоновского взаимодействия 2-x частиц является устойчивым, а его результаты совпадают с точными аналитическими решениями.

\section{Заключение}

Выполненные исследования показали, что система Galactica может использоваться для исследования 


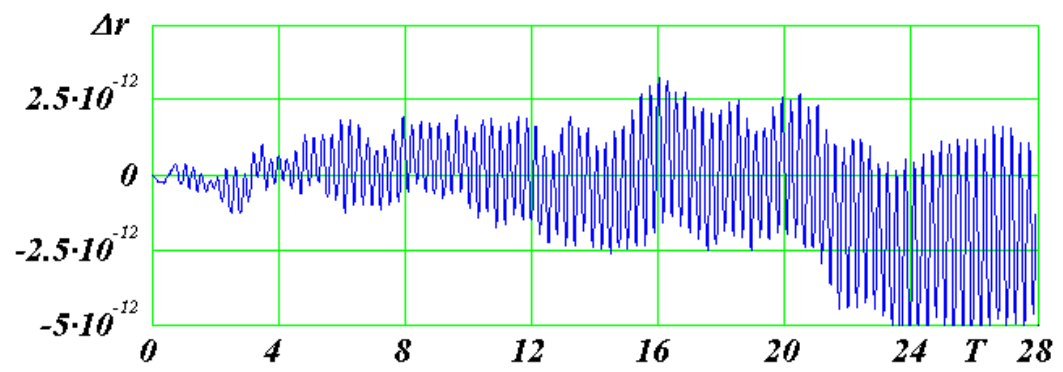

Рис 18. Изменение отклонение радиуса $\Delta r$ периферийной частицы за 100 обращений в задаче двух частиц при шаге счета $d T=1 \times 10^{-7}$.

кулоновских взаимодействий. Рассмотренная осесимметричная геометрия атома оказалась неустойчивой. Поэтому необходимо искать другие его конфигурации. Их можно получить из осесимметричной структуры поворотом орбит периферийных частиц так, чтобы они равномерно заполнили пространство. В так образованном атоме эксцентриситеты орбит немного изменяются. Однако их затем можно будет привести к необходимому значению.

Создание орбитальной модели атома потребует переосмысления многих электродинамических явлений и понятий. В первую очередь их необходимо перевести в понятия механики. В механике рассматривается воздействие одного тела на другое. Механическое воздействие на тело выражается в его ускорении. В механике это воздействие описывается силой. Часто употребляемый сленг "на тело действует сила" следует понимать: на тело действует другое тело. А сила-это наш способ описывать воздействие, которое заключается в том, как уже упоминалось, что тело приобретает ускорение.

В связи с этим электродинамическое явление, которое называют излучением, необходимо интерпретировать в механических понятиях. С позиций механики излучение не является телом, поэтому не может оказывать воздействие на другое тело. Если какое-то тело является источником излучения, то необходимо определить другое тело как приемник излучения. Только тогда можно рассматривать механическое взаимодействие источника и приемника, исключив из рассмотрения излучение. Если это не сделать, то такие взаимодействия нельзя рассматривать в рамках механики, т.к. они противоречат ее основам.

Полностью электромагнитное взаимодействие не описывается законом Кулона, т.к. оно зависит не только от расстояния между взаимодействующими частицами, но и от их относительной скорости [1]. В 20-м веке приближенное описание зависимости электромагнитного воздействия от скорости было выполнено на основе теории относительности. В ее рамках вводилась зависимость массы от скорости. Однако такая интерпретация привела к нарушению оснований механики. Поэтому появились множественные противоречия, для устранения которых вводились объекты, которые не существуют в природе. Одним из них является нейтрино [19].

Как показано в работах [20]-[22], существенное изменение траекторий частиц происходит при скоростях движения $v>0.1 c$, где $c$-скорость света. Поэтому системой Galactica с кулоновским взаимодействием можно исследовать явления до скоростей указанного порядка. Система Galactica для решения задач взаимодействия $n$-тел по закону тяготения Ньютона или закону Кулона доступна по адресу: http://www.ikz.ru/ smulski/GalactcW/. Для более высоких скоростей при кулоновских взаимодействиях необходимо использовать точное выражение для силы [1] электромагнитного взаимодействия двух частиц. В дальнейшем мы надеемся дополнить систему Galactica модулем для расчета электромагнитных взаимодействий при высоких скоростях, которые в настоящее время называются релятивистскими.

\section{References}

[1] Смульский, И.И. (1999) Теория взаимодействия. Новосибирск: Из-во Новосиб. ун-та, НИЦ ОИГГМ СО РАН, 294 p. http://www.ikz.ru/ smulski/TVfulA5_2.pdf

[2] Smulsky, J.J. (2012) Galactica Software for Solving Gravitational Interaction Problems. Applied Physics Research, 4, 110-123. http://dx.doi.org/10.5539/apr.v4n2p110 http://www.ccsenet.org/journal/index.php/apr/article/view/16773

[3] Smulsky, J.J. (2012) The System of Free Access Galactica to Compute Interactions of N-Bodies. International Journal of Modern Education and Computer Science, 4, 1-20. http://www.mecs-press.org 
http://dx.doi.org/10.5815/ijmecs.2012.11.01

[4] Google. Картинки по запросу: Planetary model of the atom.

[5] Власов, А.Д. (1993) Классическое направление в квантовой механике. М.: Московский радиотехнический институт РАН, 229 р.

[6] Kanarev, P.M. (2009) The Spectrum of the Universe. Galilean Electrodynamics, 20, 13-17.

[7] Канарёв, Ф.М. Закон формирования спектров атомов и ионов. http://www.sciteclibrary.ru/rus/catalog/pages/12586.html http://www.micro-world.su/index.php/2010-12-22-11-46-00/784-2013-01-16-02-03-51

[8] Gryziński, M. (1987) Spin-Dynamical Theory of the Wave-Corpuscular Duality. International Journal of Theoretical Physics, 26, 967-980. http://dx.doi.org/10.1007/BF00670821

[9] Gryziński, M. (1965) Classical Theory of Atomic Collisions. I. Theory of Inelastic Collisions. Physical Review A, 138, 336-358. http://dx.doi.org/10.1103/PhysRev.138.A336

[10] Gryziński, M. (1970) Ramsauer Effect as a Result of the Dynamic Structure of the Atomic Shell. Physical Review Letters, 24, 45-47. http://dx.doi.org/10.1103/PhysRevLett.24.45

[11] Gryziński, M. (1959) Classical Theory of Electronic and Ionic Inelastic Collisions. Physical Review, 115, $374-383$. http://dx.doi.org/10.1103/PhysRev.115.374

[12] Gryziński, M. (1957) Stopping Power of a Medium for Heavy, Charged Particles. Physical Review A, 107, $1471-1475$. http://dx.doi.org/10.1103/PhysRev.107.1471

[13] Яворский, Б.М. and Детлаф А.А. (1968) Справочник по физике. Для инженеров и студентов вузов. Издание четвертое, переработанное. Москва: Издательство «Наука». Главная редакция физико-математической литературы, 940 р.

[14] Смульский, И.И. (2003) Осесимметричная задача гравитационного взаимодействия N-тел//Математическое моделирование, т. 15, 27-36. http://www.smul1.newmail.ru/Russian1/IntSunSyst/Osvnb4.doc

[15] Смульский, И.И. (2008) Численное моделирование эволюции спутника вращающегося тела/В сб. Теоретические и прикладные задачи нелинейного анализа. Российская Академия Наук: ВЦ им. А.А. Дородницына. М.: ВЦ РАН А.А. Дородницына. 100-118. http://www.ikz.ru/ smulski/Papers/ModSun07c.pdf

[16] Мельников, В.П. and Смульский, И.И. (2009) Астрономическая теория ледниковых периодов: Новые приближения. Решенные и нерешенные проблемы. Новосибирск: Академическое изд-во «Гео», 2009.98 с. Книга на двух языках. С обратной стороны: Melnikov V.P., Smulsky J.J. Astronomical Theory of Ice Ages: New Approximations. Solutions and Challenges. Novosibirsk: Academic Publishing House “GEO”, 84 p. http://www.ikz.ru/ smulski/Papers/AsThAnR.pdf

[17] Мельников, В. П., Смульский, И.И. and Смульский, Я.И. (2008) Составная модель вращения Земли и возможный механизм взаимодействия континентов. Геология и Геофизика, 1129-1138. http://www.ikz.ru/ smulski/Papers/RGGRu190.pdf

[18] Smulsky, J.J. (2009) Gravitation, Field and Rotation of Mercury Perihelion. Proceedings of the 15th Annual Conference, Natural Philosophy Alliance, Albuquuerque, 7-11 April 2008, 254-260.

http://www.ikz.ru/ smulski/Papers/08Smulsky2c.pdf http://www.ikz.ru/ smulski/Papers/ModSun04.pdf

[19] Smulsky, J.J. (2012) Letter to the Antirelativists. Proceedings of the 19th Annual Conference, Natural Philosophy Alliance, Albuquerque, 25-28 July 2012, 567-568. http://www.worldsci.org/pdf/abstracts/abstracts 6667.pdf http://www.ikz.ru/ smulski/Papers/LettAntrlR.pdf

[20] Смульский, И.И. (1995) Траектории при взаимодействии двух тел, зависящем от относительного расстояния и скорости. Математическое Моделирование, 7, 117-126. http://www.smul1.newmail.ru/Russian1/FounPhisics/TrV2tl.pdf

[21] Smulsky, J.J. (2002) The New Fundamental Trajectories: Part 1. Hyperbolic/Elliptic Trajectories. Galilcan Electrodynamics, 13, 23-28. http://www.smul1.newmail.ru/English1/FounPhisics/NFT.pdf

[22] Smulsky, J.J. (2002) The New Fundamental Trajectories: Part 2. Parabolic/Elliptic Trajectories. Galilcan Electrodynamics, 13, 47-51. http://www.smul1.newmail.ru/English1/FounPhisics/NFT.pdf 
Приложение. Образец Программы В Среде Mathcad Для Создания Файла Начальных Условий

\section{In $\mathrm{CnPrClb}$.mcd. Example of calculation of the initial conditions for th program Galactica at Coulomb's interactions. Completed03.09.2012 modified 16.07.2013}

14.1. Number of bodies

$\mathrm{Nb}:=9$

$\mathrm{N}:=\mathrm{Nb}-1 \quad \mathrm{i}:=0 . . \mathrm{N} \quad \mathrm{i} 1:=1 . . \mathrm{N}$

$\mathrm{N}=8$

\subsection{Constants}

From Handbook by Yavorsky \& Detlaf: pp.749, 910, 912, 913.

$$
\begin{aligned}
& \mathrm{m}_{\mathrm{e}}:=9.1091 \cdot 10^{-31} \mathrm{~m}_{\mathrm{p}}:=1.67252 \cdot 10^{-27} \quad \mathrm{~m}_{\mathrm{ne}}:=1.67482 \cdot 10^{-27} \quad \mathrm{e}_{\mathrm{e}}:=4.80298 \cdot 10^{-10} \\
& \mathrm{R} 0:=1.5 \cdot 10^{-15} \quad \mathrm{R}_{\mathrm{e}}:=1.5 \cdot 10^{-15} \quad{ }^{\mathrm{a}}{ }_{\mathrm{B} 0}:=5.29167 \cdot 10^{-11} \quad \varepsilon \mathrm{d}:=1
\end{aligned}
$$

14.3. Properties of bodies and of their motions

$$
\begin{aligned}
& \mathrm{A}_{\mathrm{N}}:=16 \quad \mathrm{Z}_{\mathrm{n}}:=8 \quad \mathrm{n}_{\mathrm{n}}:=2 \quad \mathrm{l}_{\mathrm{n}}:=1 \quad \mathrm{r}_{\mathrm{m}}:=\mathrm{a} \mathrm{B} 0 \cdot \frac{\left[3 \cdot \mathrm{n}_{\mathrm{n}}^{2}-\mathrm{l}_{\mathrm{n}} \cdot\left(\mathrm{l}_{\mathrm{n}}+1\right)\right]}{2 \cdot \mathrm{Z}_{\mathrm{n}}} \quad \mathrm{r}_{\mathrm{n}}:=\mathrm{R} 0 \cdot \mathrm{A}_{\mathrm{N}}{ }^{\frac{1}{3}} \\
& \mathrm{a}:=\mathrm{r}_{\mathrm{m}} \quad \mathrm{e}:=0.15 \quad \varepsilon 0:=0.409 \quad \mathrm{a}=3.30729375 \cdot 10^{-11} \quad \mathrm{r}_{\mathrm{n}}=3.77976314968462 \cdot 10^{-15}
\end{aligned}
$$

14.4. Masses of bodies in $\mathrm{kg}$ and their radiuses in $\mathrm{m}$

$$
\mathrm{m}_{0}:=\mathrm{Z}_{\mathrm{n}} \cdot \mathrm{m}_{\mathrm{p}}+\mathrm{Z}_{\mathrm{n}} \cdot \mathrm{m}_{\mathrm{ne}} \quad \mathrm{m}_{\mathrm{i} 1}:=\mathrm{m}_{\mathrm{e}} \quad \operatorname{ram}_{0}:=\mathrm{r}_{\mathrm{n}} \quad \operatorname{ram}_{\mathrm{i} 1}:=\mathrm{R}_{\mathrm{e}}
$$

14.5. Coordinates and velocities of bodies acordindly to:

Smulsky J.J. Axisymmetrical problem of gravitational interaction of N-bodies // Mathematical modelling. 2003, Vol. 15, No 5, Pp. 27-36. (In Russian http:/www.smul1.newmail.ru/Russian1/IntSunSyst/Osvnb4.doc).

For two bodies interactions see item 14.14.

$$
\begin{aligned}
& \alpha_{1}:=-\frac{1}{1+\mathrm{e}} \quad \mathrm{Rp}:=\mathrm{a} \cdot \frac{2 \cdot \alpha_{1}+1}{\alpha_{1}} \quad \mathrm{f}_{\mathrm{N}}:=0.25 \cdot \sum_{\mathrm{i} 2=2}^{\mathrm{N}} \frac{1}{\sin \left[\frac{(\mathrm{i} 2-1) \cdot \pi}{\mathrm{N}}\right]} \\
& \mu 1:=-\frac{10^{-9} \cdot \mathrm{e}^{2}}{\mathrm{~m}_{1} \cdot \varepsilon \mathrm{d}} \cdot\left(\mathrm{N}-\mathrm{f}_{\mathrm{N}}\right) \\
& \mathrm{vp}:=\sqrt{\frac{\mu 1}{\alpha_{1} \cdot \mathrm{Rp}} \quad \mathrm{d} \phi:=\frac{2 \cdot \pi}{\mathrm{N}} \quad \phi 0_{\mathrm{i} 1}:=(\mathrm{i} 1-1) \cdot \mathrm{d} \phi} \quad \alpha_{1}=-0.869565217391304
\end{aligned}
$$

Coordinates and velocities in the plane of orbits, in $\mathrm{m}$ and $\mathrm{m} / \mathrm{sec}$

$$
\mathrm{xo}_{\mathrm{i} 1}:=\mathrm{Rp} \cdot \cos \left(\phi 0_{\mathrm{i} 1}\right) \quad \mathrm{yo}_{\mathrm{i} 1}:=\mathrm{Rp} \cdot \sin \left(\phi 0_{\mathrm{i} 1}\right) \quad \mathrm{zo}_{\mathrm{i} 1}:=0 \quad \mu 1=-1.315657523093202 \cdot 10^{3}
$$


$\mathrm{vxo}_{\mathrm{i} 1}:=-\mathrm{vp} \cdot \sin \left(\phi 0_{\mathrm{i} 1}\right) \quad \mathrm{vyo}_{\mathrm{i} 1}:=\mathrm{vp} \cdot \cos \left(\phi 0_{\mathrm{i} 1}\right) \quad \mathrm{vzo}_{\mathrm{i} 1}:=0 \quad \mathrm{f}_{\mathrm{N}}=2.804865846209121$

Checking the distanses between bodies. If $d$ is less than 50 , it is need to increase a. do $:=\sqrt{\left(\mathrm{xo}_{2}-\mathrm{xo}_{1}\right)^{2}+\left(\mathrm{yo}_{2}-\mathrm{yo}_{1}\right)^{2}+\left(\mathrm{zo}_{2}-\mathrm{zo}_{1}\right)^{2}} \quad \mathrm{~d}:=\frac{\mathrm{do}}{\left(2 \cdot \mathrm{ram}_{1}\right)} \quad \mathrm{d}=7.171996969841117 \cdot 10^{3}$

14.6. The exact motion of bodies. Checking the planned configuration of the probl

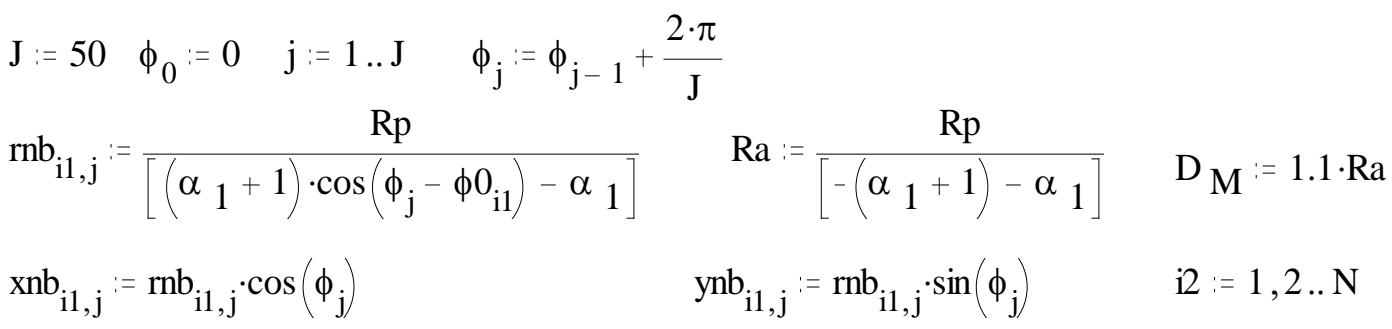

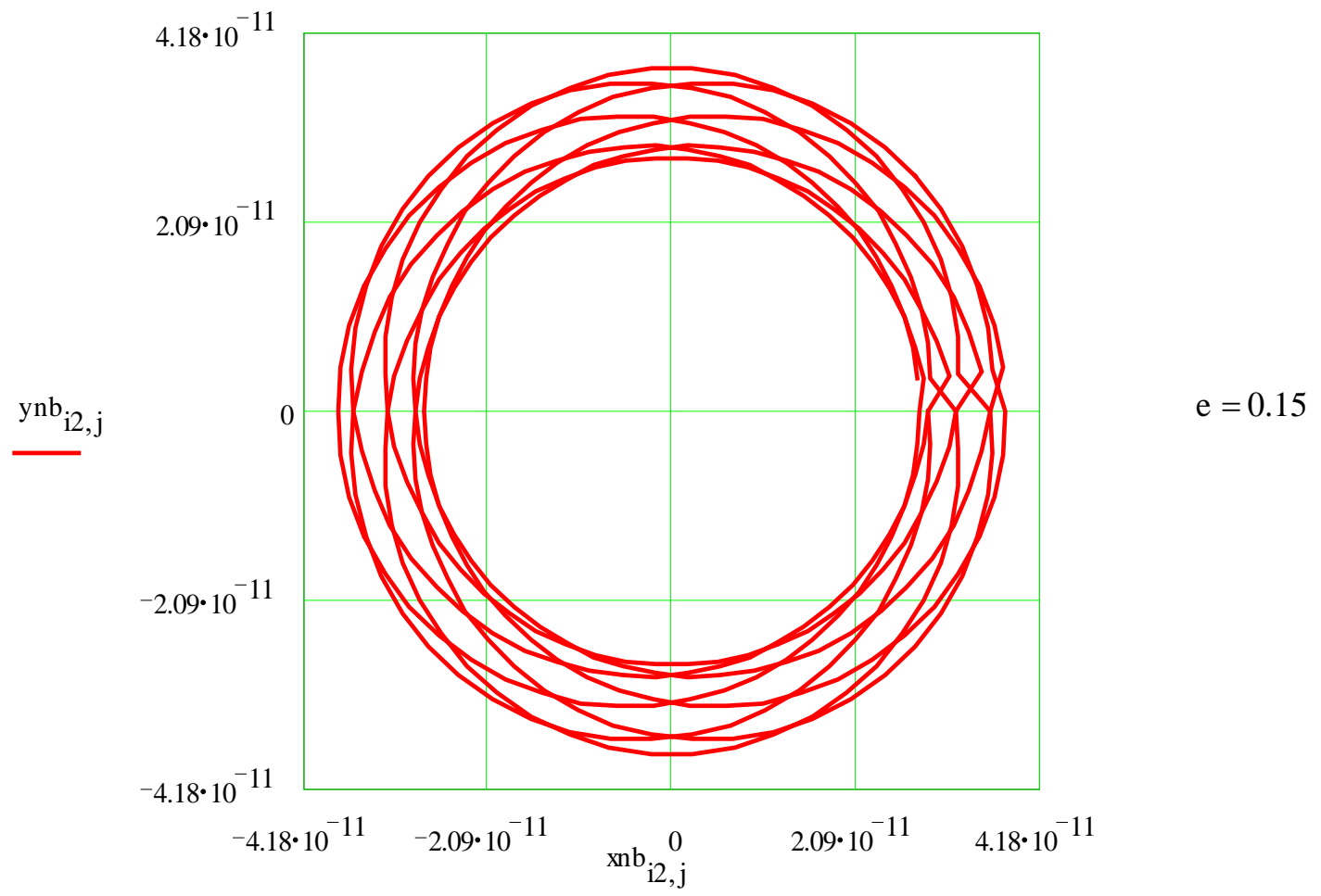

\subsection{Coordinates and velocities in the equatorial plane}

$$
\begin{array}{llll}
\mathrm{xm}_{\mathrm{i} 1}:=\mathrm{xo}_{\mathrm{i} 1} \quad \mathrm{ym}_{\mathrm{i} 1}:=\mathrm{yo}_{\mathrm{i} 1} \cdot \cos (\varepsilon 0)-\mathrm{zo}_{\mathrm{i} 1} \cdot \sin (\varepsilon 0) & \mathrm{zm}_{\mathrm{i} 1}:=\mathrm{yo}_{\mathrm{i} 1} \cdot \sin (\varepsilon 0)+\mathrm{zo}_{\mathrm{i} 1} \cdot \cos (\varepsilon 0) \\
\mathrm{vxms}_{\mathrm{i} 1}:=\mathrm{vxo}_{\mathrm{i} 1} \mathrm{vyms}_{\mathrm{i} 1}:=\mathrm{vyo}_{\mathrm{i} 1} \cdot \cos (\varepsilon 0)-\mathrm{vzo}_{\mathrm{i} 1} \cdot \sin (\varepsilon 0) \quad \mathrm{vzms}_{\mathrm{i} 1}:=\mathrm{vyo}_{\mathrm{i} 1} \cdot \sin (\varepsilon 0)+\mathrm{vzo}_{\mathrm{i} 1} \cdot \cos (\varepsilon 0) \\
\mathrm{xm}_{0}:=0 \quad \mathrm{ym}_{0}:=0 \quad \quad \mathrm{zm}_{0}:=0 \quad \mathrm{vxms}_{0}:=0 \quad \mathrm{vyms}_{0}:=0 \quad \mathrm{vzms}_{0}:=0
\end{array}
$$




\subsection{The transition to the dimensionless variables}

$$
\begin{array}{lll}
\mathrm{ms}_{0}:=0 & \mathrm{~ms}_{\mathrm{i}+1}:=\mathrm{ms}_{\mathrm{i}}+\mathrm{m}_{\mathrm{i}} & \mathrm{mo}_{\mathrm{i}}:=\frac{\mathrm{m}_{\mathrm{i}}}{\mathrm{ms}_{\mathrm{N}+1}}
\end{array} \quad \mathrm{P}_{\mathrm{M}}:=1 \cdot 10^{-15}
$$

Determination of the lenath scale that the dimensionless time unit is equel one $10^{\wedge}-15$ of $s$ $\mathrm{k}_{\mathrm{t}}:=\frac{1}{\mathrm{P}_{\mathrm{M}}} \quad \mathrm{Am}:=\left[\frac{\left(10^{-9} \cdot \mathrm{e}_{\mathrm{e}}^{2}\right)}{\varepsilon \mathrm{d} \cdot \mathrm{ms}_{\mathrm{N}+1} \cdot \mathrm{k}_{\mathrm{t}}^{2}}\right]^{\frac{1}{3}} \quad \mathrm{Am}=2.049767130503777 \cdot 10^{-11}$

\section{Clarification of Am}

$$
\begin{aligned}
& \operatorname{Am}:=2.049767130503774 \cdot 10^{-11} \quad \mathrm{k}_{\mathrm{v}}:=\sqrt{\frac{\varepsilon \mathrm{d} \cdot \mathrm{ms}_{\mathrm{N}+1} \cdot \mathrm{Am}}{10^{-9} \cdot \mathrm{e}^{2}}} \quad \mathrm{Am}:=\frac{1}{\mathrm{k}_{\mathrm{t}} \cdot \mathrm{k}_{\mathrm{v}}} \\
& \mathrm{x}_{\mathrm{i}}:=\frac{\mathrm{xm}_{\mathrm{i}}}{\mathrm{Am}} \quad \mathrm{y}_{\mathrm{i}}:=\frac{\mathrm{ym}_{\mathrm{i}}}{\mathrm{Am}} \quad \mathrm{z}_{\mathrm{i}}:=\frac{\mathrm{zm}_{\mathrm{i}}}{\mathrm{Am}} \quad \mathrm{ra}_{\mathrm{i}}:=\frac{\mathrm{ram}_{\mathrm{i}}}{\mathrm{Am}} \quad \mathrm{Am}=2.049767130503774 \cdot 10-11 \\
& \mathrm{vx}_{\mathrm{i}}:=\mathrm{vxms}_{\mathrm{i}} \cdot \mathrm{k}_{\mathrm{v}} \quad \mathrm{vy}_{\mathrm{i}}:=\mathrm{vyms}_{\mathrm{i}} \cdot \mathrm{k}_{\mathrm{v}} \quad \mathrm{vz}_{\mathrm{i}}:=\mathrm{vzms}_{\mathrm{i}} \cdot \mathrm{k}_{\mathrm{v}}
\end{aligned}
$$

14.9. The center of mass of the interacting bodies

$$
\begin{array}{llll}
\mathrm{Xc}_{0}:=0 & \mathrm{Yc}_{0}:=0 \quad \mathrm{Zc}_{0}:=0 & \mathrm{Vxc}_{0}:=0 \quad \mathrm{Vyc}_{0}:=0 \quad \mathrm{Vzc}_{0}:=0 \\
\mathrm{Xc}_{\mathrm{i}+1}:=\mathrm{Xc}_{\mathrm{i}}+\mathrm{mo}_{\mathrm{i}} \cdot \mathrm{x}_{\mathrm{i}} & \mathrm{Yc}_{\mathrm{i}+1}:=\mathrm{Yc}_{\mathrm{i}}+\mathrm{mo}_{\mathrm{i}} \cdot \mathrm{y}_{\mathrm{i}} & \mathrm{Zc}_{\mathrm{i}+1}:=\mathrm{Zc}_{\mathrm{i}}+\mathrm{mo}_{\mathrm{i}} \cdot \mathrm{z}_{\mathrm{i}} \\
\mathrm{Vxc}_{\mathrm{i}+1}:=\mathrm{Vxc}_{\mathrm{i}}+\mathrm{mo}_{\mathrm{i}} \cdot \mathrm{Vx}_{\mathrm{i}} & \mathrm{Vyc}_{\mathrm{i}+1}:=\mathrm{Vyc}_{\mathrm{i}}+\mathrm{mo}_{\mathrm{i}} \cdot \mathrm{Vy}_{\mathrm{i}} \quad \mathrm{Vzc}_{\mathrm{i}+1}:=\mathrm{Vzc}_{\mathrm{i}}+\mathrm{mo}_{\mathrm{i}} \cdot \mathrm{Vz}_{\mathrm{i}}
\end{array}
$$

Coordinates and velocities relatively the center of mass

$$
\begin{array}{llll}
\mathrm{x}_{\mathrm{i}}:=\mathrm{x}_{\mathrm{i}}-\mathrm{Xc}_{\mathrm{N}+1} & \mathrm{y}_{\mathrm{i}}:=\mathrm{y}_{\mathrm{i}}-\mathrm{Yc}_{\mathrm{N}+1} & \mathrm{z}_{\mathrm{i}}:=\mathrm{z}_{\mathrm{i}}-\mathrm{Zc}_{\mathrm{N}+1} \quad \mathrm{Xc}_{\mathrm{N}+1}=-2.032879073410321 \cdot 10^{-20} \\
\mathrm{vx}_{\mathrm{i}}:=\mathrm{vx}_{\mathrm{i}}-\mathrm{Vxc}_{\mathrm{N}+1} & \mathrm{vy}_{\mathrm{i}}:=\mathrm{vy}_{\mathrm{i}}-\mathrm{Vyc}_{\mathrm{N}+1} & \mathrm{vZ}_{\mathrm{i}}:=\mathrm{vZ}_{\mathrm{i}}-\mathrm{Vzc}_{\mathrm{N}+1} \mathrm{Vxc}_{\mathrm{N}+1}=5.204170427930421 \cdot 10^{-18}
\end{array}
$$

\subsection{Angular momentum of the system of bodies}

$$
\begin{aligned}
& \mathrm{Mx}_{\mathrm{i}}:=0 \quad \mathrm{My}_{\mathrm{i}}:=0 \quad \mathrm{Mz}_{\mathrm{i}}:=0
\end{aligned}
$$

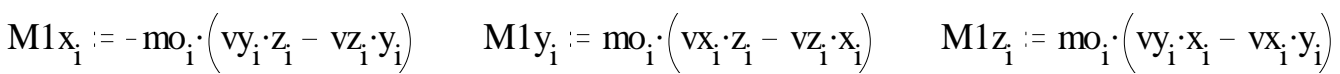

$$
\begin{aligned}
& \mathrm{Mx}_{\mathrm{i}+1}:=\left(\mathrm{Mx}_{\mathrm{i}}\right)+\mathrm{M} 1 \mathrm{x}_{\mathrm{i}} \quad \mathrm{My}_{\mathrm{i}+1}:=\left(\mathrm{My}_{\mathrm{i}}\right)+\mathrm{M} 1 \mathrm{y}_{\mathrm{i}} \quad \mathrm{Mz}_{\mathrm{i}+1}:=(\mathrm{Mz})+\mathrm{M} 1 \mathrm{z}_{\mathrm{i}} \\
& \mathrm{Mx}_{\mathrm{N}+1}=-9.665352161902836 \cdot 10^{-19} \mathrm{My}_{\mathrm{N}+1}=-0.053108187925464 \\
& \mathrm{Mz}_{\mathrm{N}+1}=0.122526400051667
\end{aligned}
$$




\section{Verification}

$$
\begin{aligned}
& \mathrm{msr}_{0}:=0 \quad \mathrm{msr}_{\mathrm{i}+1}:=\mathrm{msr}_{\mathrm{i}}+\mathrm{mo}_{\mathrm{i}} \quad \mathrm{msr}_{\mathrm{N}+1}=1 \\
& \mathrm{M} 00:=\frac{\mathrm{N} \cdot \mathrm{mo}_{1} \cdot \mathrm{Rp} \cdot \mathrm{vp} \cdot \mathrm{k}_{\mathrm{v}}}{\mathrm{Am}} \quad \mathrm{M} 0:=\sqrt{\left(\mathrm{Mx}_{\mathrm{N}+1}\right)^{2}+\left(\mathrm{My}_{\mathrm{N}+1}\right)^{2}+\left(\mathrm{Mz}_{\mathrm{N}+1}\right)^{2}} \\
& \frac{\mathrm{M} 0-\mathrm{M} 00}{\mathrm{M} 0}=0 \quad\left(\mathrm{Am}-\frac{1}{\mathrm{k}_{\mathrm{t}} \cdot \mathrm{k}_{\mathrm{v}}}\right) \cdot \frac{1}{\mathrm{Am}}=0
\end{aligned}
$$

14.11. To coordinate the size of area with the number of bodies need to change the $B<=\mathbf{1}$ and $\mathrm{C} 1<=1$ so that $\mathrm{Nbar}>=\mathrm{Nb}$.

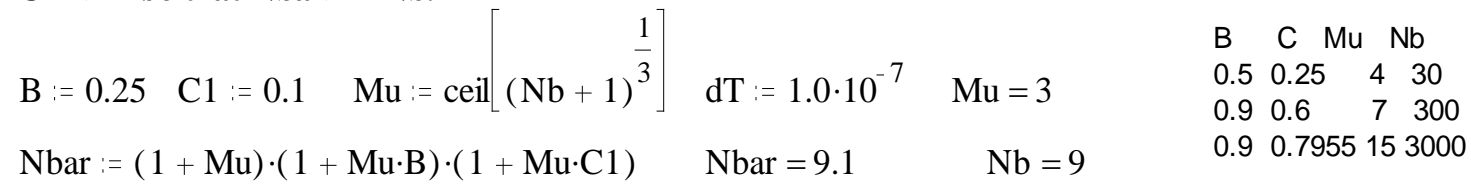

\subsection{Writing data to the file name.prn}

$$
\begin{aligned}
& \mathrm{k}:=0 . .14 \quad \mathrm{l}:=7 . .12 \quad \mathrm{l} 2:=5 . .14 \quad \mathrm{R}_{0, \mathrm{k}}:=0 \quad \mathrm{R}_{0,7}:=\mathrm{Mx}_{\mathrm{N}+1} \quad \mathrm{R}_{0,8}:=\mathrm{My}_{\mathrm{N}+1} \\
& \mathrm{R}_{0,9}:=\mathrm{Mz}_{\mathrm{N}+1} \quad \mathrm{R}_{1,0}:=0 \quad \mathrm{R}_{1,1}:=\mathrm{dT} \quad \mathrm{R}_{1,2}:=1 \quad \mathrm{R}_{1,3}:=1 \quad \mathrm{R}_{1,4}:=\mathrm{Nb} \\
& \mathrm{R}_{1,5}:=1 \quad \mathrm{R}_{1,6}:=\mathrm{B} \quad \mathrm{R}_{1,7}:=\mathrm{C} 1 \quad \mathrm{R}_{1,8}:=\mathrm{Mu} \quad \mathrm{R}_{1,9}:=\varepsilon \mathrm{d} \quad \mathrm{R}_{\mathrm{i}+2,0}:=\operatorname{mo}_{\mathrm{i}} \quad \mathrm{R}_{\mathrm{i}+2,1}:=\mathrm{x}_{\mathrm{i}} \\
& \mathrm{R}_{\mathrm{i}+2,2}:=\mathrm{y}_{\mathrm{i}} \quad \mathrm{R}_{\mathrm{i}+2,3}:=\mathrm{z}_{\mathrm{i}} \quad \mathrm{R}_{\mathrm{i}+2,4}:=\mathrm{vx}_{\mathrm{i}} \quad \mathrm{R}_{\mathrm{i}+2,5}:=\mathrm{vy}_{\mathrm{i}} \quad \mathrm{R}_{\mathrm{i}+2,6}:=\mathrm{vz}_{\mathrm{i}} \quad \mathrm{R}_{\mathrm{i}+2,1}:=0 \\
& \mathrm{R}_{\mathrm{i}+2,13}:=\mathrm{ra}_{\mathrm{i}} \quad \mathrm{R}_{\mathrm{i}+2,14}:=0 \quad \mathrm{R}_{\mathrm{i}+2,15}:=\mathrm{qo}_{\mathrm{i}}
\end{aligned}
$$

Information line at end of file $\mathrm{B}:=\mathrm{Nb}+2$



$$
\text { WwwwRITEPRN("axsykl09.prn") := R e } 0.15 \quad 0
$$

$$
\text { "axsyk109.prn" "axsykc09.prn" }
$$

It is necessary 1 zero at the end of 1-nd line and 6 zero at the end of 2-nd line of a file name.pm to remove and to rename the file into a name.dat.

Verification coordinates and velocities
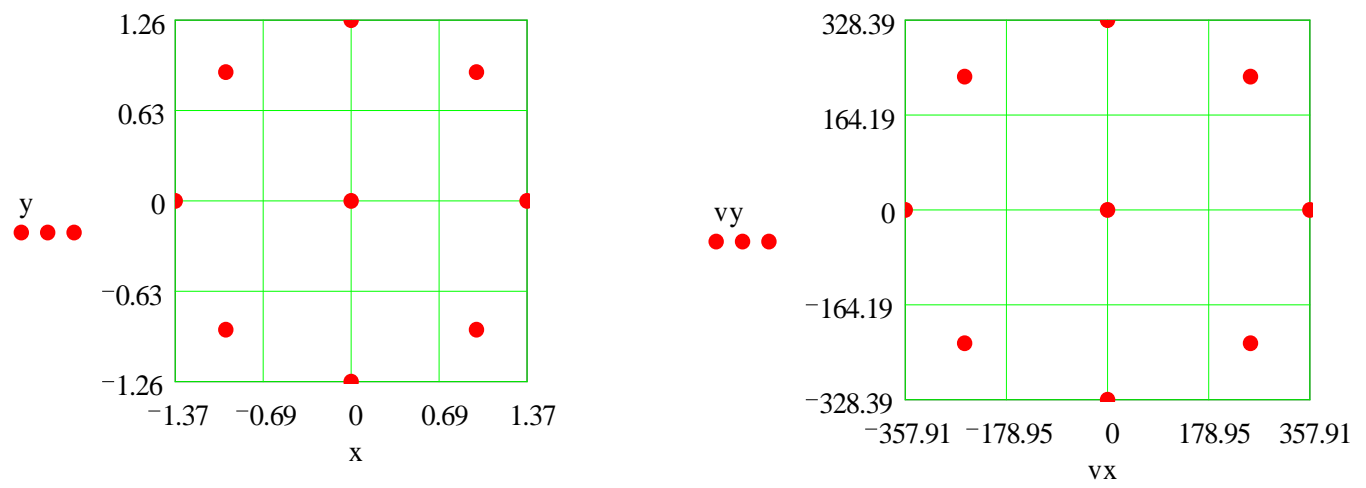


\subsection{Reading the file of type garek29.dat}

$$
\begin{aligned}
& \text { Nbg := } 9 \quad \mathrm{Ng}:=\mathrm{Nbg}-1 \quad \text { ig }:=0 . . \mathrm{Ng} \quad \text { ig2 }:=0 . .24+\mathrm{Nbg} \cdot 16 \\
& \text { P2 }{ }_{\text {ig2 }}:=\text { READ( "garek29.dat") "garek29.dat" }
\end{aligned}
$$

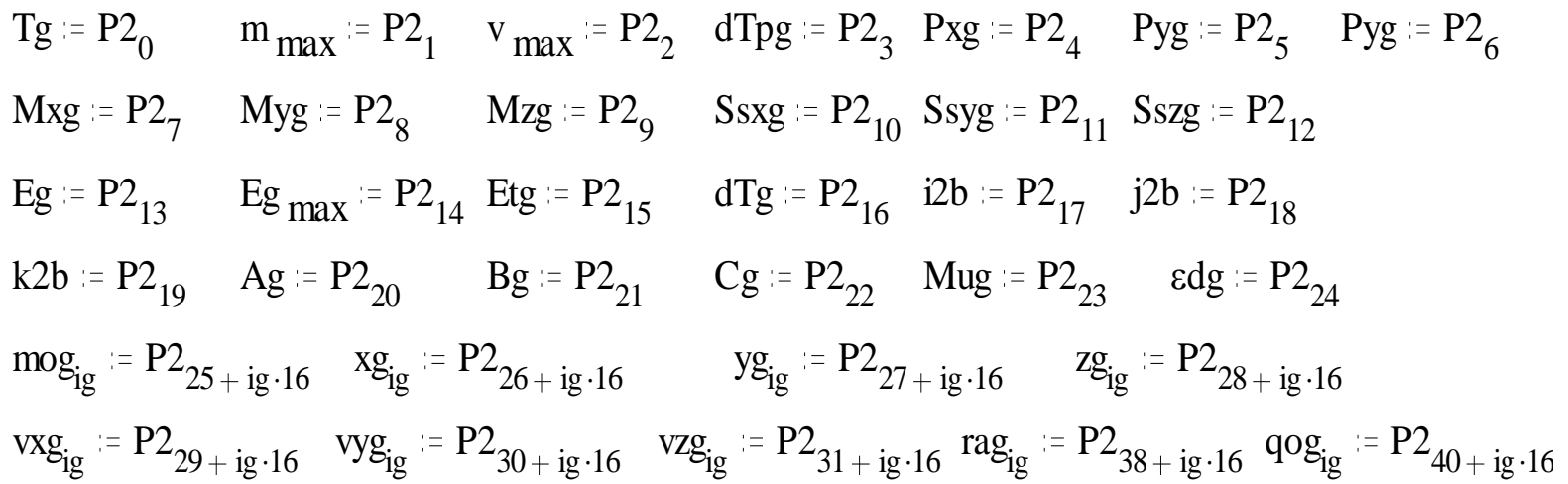$$
\operatorname{Tg}=1 \cdot 10^{-10}
$$

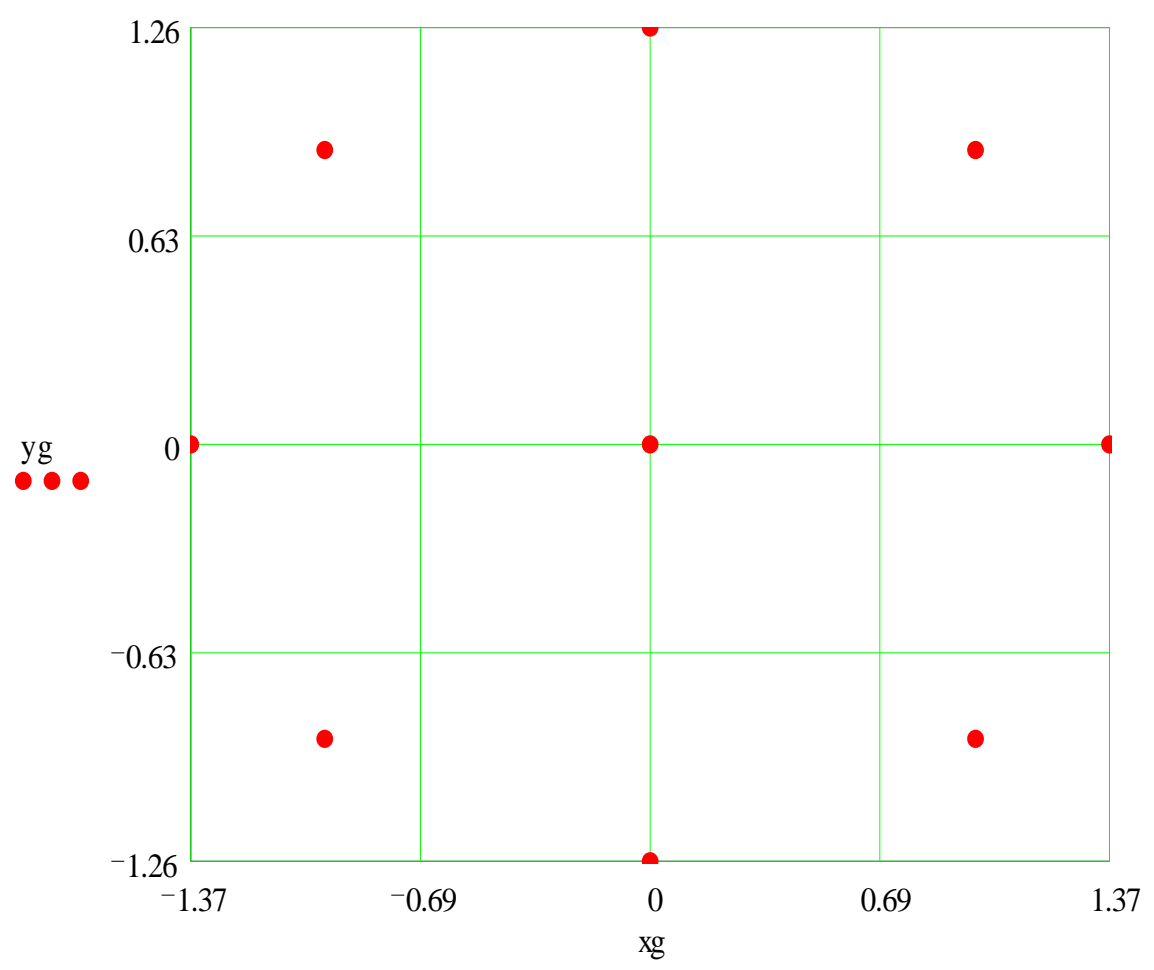

14.14. Change in item 14.5 for two bodies interactions
$\alpha_{1}:=-\frac{1}{1+e}$
$\operatorname{Rp}:=a \cdot \frac{2 \cdot \alpha_{1}+1}{\alpha_{1}}$
$\mathrm{m}_{\mathrm{pr}}:=\frac{\mathrm{m}_{0} \cdot \mathrm{m}_{1}}{\mathrm{~m}_{0}+\mathrm{m}_{1}}$
$\mu 1:=-\left(\frac{10^{-9} \cdot \mathrm{e}^{2}}{\mathrm{~m}_{\mathrm{pr}} \cdot \varepsilon \mathrm{d}}\right)$ 